\title{
Stress Propagation and Arching in Static Sandpiles
}

\author{
J. P. Wittmer and M. E. Cates \\ Department of Physics and Astronomy \\ University of Edinburgh, JCMB King's Buildings \\ Mayfield Road, Edinburgh EH9 3JZ, UK \\ P. Claudin* \\ Cavendish Laboratory, Madingley Road \\ Cambridge CB3 OHE, UK \\ * Present Address: \\ Service de Physique de l'Etat Condensé, CEA \\ Ormes des Merisiers, 91191 Gif-sur-Yvette, Cedex France
}

PACS numbers 46.10.+z, 46.30.-i, 81.35.+k, 83.70 Fn 


\begin{abstract}
We present a new approach to the modelling of stress propagation in static granular media, focussing on the conical sandpile constructed from a point source. We view the medium as consisting of cohesionless hard particles held up by static frictional forces; these are subject to microscopic indeterminacy which corresponds macroscopically to the fact that the equations of stress continuity are incomplete - no strain variable can be defined. We propose that in general the continuity equations should be closed by means of a constitutive relation (or relations) between different components of the (mesoscopically averaged) stress tensor. The primary constitutive relation relates radial and vertical shear and normal stresses (in two dimensions, this is all one needs). We argue that the constitutive relation(s) should be local, and should encode the construction history of the pile: this history determines the organization of the grains at a mesoscopic scale, and thereby the local relationship between stresses. To the accuracy of published experiments, the pattern of stresses beneath a pile shows a scaling between piles of different heights (RSF scaling) which severely limits the form the constitutive relation can take; various asymptotic features of the stress patterns can be predicted on the basis of this scaling alone. To proceed further, one requires an explicit choice of constitutive relation; we review some from the literature and present two new proposals. The first, the FPA (fixed principal axes) model, assumes that the eigendirections (but not the eigenvalues) of the stress tensor are determined forever when a material element is first buried. (This assumes, among other things, that subsequent loadings are not so large as to produce slip deep inside the pile.) A macroscopic consequence of this mesoscopic assumption is that the principal axes have fixed orientation in space: the major axis everywhere bisects the vertical and the free surface. As a result of this, stresses propagate along a nested set of archlike structures within the pile, resulting in a minimum of the vertical normal stress between the apex of the pile, as seen experimentally ("the dip"). This experiment has not been explained within previous continuum approaches; the appearance of arches within our model corroborates earlier physical arguments (of S. F. Edwards and others) as to the origin of the dip, and places them on a more secure mathematical footing. The second model is that of "oriented stress linearity" (OSL) which contains an adjustable parameter (one value of which corresponds to FPA). For the general OSL case, the simple interpretation in terms of nested arches does not apply, though a dip is again found over a finite parameter range. In three dimensions, the choice for the primary constitutive relation must be supplemented by a secondary one; we have tried several, and find that the results for the stresses in a three dimensional (conical) pile do not depend much on which secondary closure is chosen. Three dimensional results for the FPA model are in good semiquantitative agreement with published experimental data on conical piles (including the dip); the data does not exclude, but nor does it support, OSL parameters somewhat different from FPA. The modelling strategy we adopt, based on local, history-dependent constitutive relations among stresses, leads to nontrivial predictions for piles which are prepared with a different construction history from the normal one. We consider several such histories in which a pile is prepared and parts of it then removed and/or tilted. Experiments along these lines could provide a searching test of the theory.
\end{abstract}




\section{Introduction}

A sandpile is normally constructed by pouring sand from a stationary point source, as shown in Fig.1(a). Each element of sand arrives at the apex of the pile, rolls down the slopes, comes to rest, and is finally buried. The final (static) sandpile then consists of a symmetrical cone whose surface is at the angle of repose of the material. Some of the simplest questions one can ask about this system concern the distribution of stresses in the pile. Specifically, it is possible experimentally to measure the downward force on the supporting surface at different positions under the pile [1, 2]. (Throughout the paper we assume this to be a high friction surface so that slip does not occur at it.) Intuitively one can guess that the maximum force would be recorded directly beneath the apex of the pile; but in fact, the experiments show a pronounced dip in the force beneath the apex. This counterintuitive result has stimulated various theoretical [3, 4, 5, 6, 7] and computational [8, 9] studies; but so far, there has been no clear consensus on the origin of the dip.

In the present work we pursue a continuum mechanics approach based on the equations of stress continuity in a cohesionless granular medium [10, 7, 11, 12]. This approach immediately encounters the problem of indeterminacy: even in the simplest case of a two-dimensional pile (which we consider in detail), the continuity of stress does not lead to a closed set of equations. In elastic materials, this deficiency would be rectified by invoking the usual constitutive relation between stress and strain (Hooke's law). For granular media, however, there is no clear definition of "strain". Rather, it is widely assumed that the physics of granular media can be understood purely in terms of rigid particles packed together in frictional contact (so that no strain variables can be defined). The indeterminacy of the stress equations then has a clear origin: for two rigid particles in frictional contact with a specified normal force, the coefficient of static friction defines only the maximum shear force that may be present. Our continuum mechanics approach, like some previous ones [7] assumes that, despite this local indeterminacy, there emerges on length scales much larger than the grain size some definite relation between the average frictional and normal forces. Thus we assume the existence of one or more constitutive relations, not between stress and strain, but among the various components of the stress tensor itself. In two dimensions, one such relation is enough to close the equations; in three dimensions (subject to certain symmetry assumptions) two are needed.

A basic tenet of our approach is that the constitutive relations are local: we assume that that these relations between stresses do not depend on distant perturbations, although the stresses themselves certainly do. Clearly, the constitutive relation (or relations - we suppress the plural in what follows), between stresses in some material element, must reflect the packing arrangement of grains in that element. This raises the possibility that the constitutive behaviour could vary from place to place in the pile. More generally, we believe that in principle the constitutive relation of a given material element should encode its entire construction history. We shall assume, however, that the important part of this history comes to an end at the moment where the element is buried: at later times, although the stresses passing through the element may vary, the constitutive relation between them cannot. We call this the assumption of perfect memory. We show below (Sec. 2.3) that the perfect memory and locality assumptions, when combined with a simple and experimentally motivated scaling assumption (called 
RSF scaling), drastically limit the form the constitutive equation can take.

A consequence of perfect memory is that the stresses in an element, once buried, respond reversibly (though not necessarily linearly) to any subsequent additional loading. Such loadings can be brought about either by adding more material to the pile, or by putting a small weight on its surface, for example. Obviously our perfect memory assumption, and indeed that of locality, may fail if the load added is so large as to lead to rearrangement of grains within the element (that is, slip). ¿From our viewpoint, however, if slip does occur, this represents a change in construction history which must explicitly be taken into account. It turns out that for most of the models and geometries considered in this paper, perturbative loadings of the pile do not, in fact, cause slip except at the surface of the pile.

Our assumption of a local, history-dependent constitutive relation among stresses is not widely accepted as a modelling strategy for sandpiles. (Indeed, we have not seen any really clear exposition of this strategy in the previous literature.) Many would argue the necessity of explicitly invoking the deformability of particles (allowing a strain variable to enter); others would argue that infinitesimal distant loads should cause rearrangement of a network of contacts among hard particles (leading to intrinsically nonlocal stress propagation). This paper aims to explore in detail the kinds of prediction that can be made within our overall modelling strategy, and to introduce some physically plausible candidates for constitutive relations.

\subsection{Previous continuum approaches}

Much existing work on the static continuum mechanics of cohensionless granular materials has invoked, as an implicit constitutive relation, the assumption of Incipient Failure Everywhere (IFE). That this is indeed an assumption, is not always made clear in the engineering literature [10, 11, 12, 13]. The IFE model supposes that the material is everywhere just on the point of slip failure: all frictional forces are "fully mobilized". Thus an appropriately chosen (local) yield criterion [10, 12] of the material provides the missing constitutive equation.

The physics of this assumption is dubious. When a pile is made from a point source (Fig.1(a)) there is a continuous series of landslides at the surface; we therefore accept that an incipient failure condition is maintained at the surface. Even in saying this, we ignore the distinction between the angle of repose (that of the free surface just after a landslide) and the maximum angle of stability (that just before). These differ by the Bagnold hysteresis angle, which is small compared to the repose angle itself [14, 15]; we neglect this hysteresis effect from now on. However, the validity of the incipient failure condition at the surface, which contains material elements just at the point of burial, does not mean that the same condition still holds for an element long afterwards. Such an element lies deep beneath the surface, and has since burial been loaded by adding more material to the pile above. In fact, the IFE assumption can clearly be ruled out on experimental grounds: as we show below, it fails to account for the dip.

The IFE closure implies that two of the principal stresses are proportional [10]. In contrast, proportionality of the horizontal and vertical normal stresses was proposed as a constitutive relation recently by Bouchaud, Cates and Claudin [7] (we refer to this closure as the BCC model). In two dimensions it was found that the stress continuity equation then has a convenient analytic property: it becomes a wave equation. However 
[7], the BCC model predicts a stress plateau, rather than a dip, at the centre of a sandpile. Attempts to explain the dip by introducing various nonlinear terms in the constitutive relation proved unconvincing, at least for small nonlinearities, whose perturbative inclusion showed a hump instead of a dip. The BCC paper (Sec.3.2 of Ref. [7]) in fact included a brief discussion of certain strongly nonlinear models [16], which were also argued to give a hump. This conclusion turns out to be incorrect, for subtle reasons that we discuss below (Sec. 2.9). In one sense, the "oriented stress linearity" (OSL) model, which we study in detail in this paper, can be viewed as an extreme limit of this type of model. The OSL model, like BCC, has a linear relation between normal stresses but now in a coordinate system that is tilted (through a constant angle) with respect to the vertical.

An apparently completely different approach to describing the stress distribution in sandpiles was proposed by Edwards and Oakeshott [5]. These authors considered a pile consisting of a stack of nested arches, Fig.1(b). (A recent modification considers a model of platelike granules and allows curvature of the arches [6]). Each arch supports only its own weight, and consequently the vertical stress decreases for the smaller arches near the centre of the pile. This approach provides a very appealing physical picture of why there is a dip. In the arching mechanism, the load in an element is transmitted unevenly to those below. The central part of the pile is thereby "screened" from additional loadings which are supported instead by the outer regions.

However, there are some obvious drawbacks with this approach. Firstly, the dip is greatly overpredicted: by construction there is no downward force whatever at the centre of the pile, while experiments show a finite value. Secondly, unless the arches are parallel to the free surface, the outermost "arches" are incomplete. It is mechanically impossible for one of these to transmit its weight purely along its own length: there is an unbalanced couple about the base of such an arch which would cause it to fall over. If, instead, the arches are parallel to the free surface then the model is stable, but it predicts an abrupt discontinuity in the downward force at the edge of the pile, which is not observed.

These difficulties arise, at least in part, from an inconsistent attempt to treat the vertical normal stress ("weight") independently of the other stress components. This is rectified in the OSL models introduced below. Among these is a special case, the "fixed principal axes" (FPA) model, which is very close in its physical content to the picture of nested arches originally suggested by Edwards and Oakeshott. As its name suggests, in the FPA model, the principal axes of the stress tensor have a constant angle of inclination to the vertical. These axes turn out to coincide with the stresspropagation characteristics, which resemble a set of Edwards arches (Sec. 2.7). In fact, we believe that our FPA model gives, for the first time, a fully consistent continuum mechanics implementation of Edwards' arching picture. As shown in Sec. 3, this model gives good agreement with experimental data in three-dimensional sandpiles.

The discussion above (like others in the recent physics literature on sandpiles) attributes the idea of arching to Edwards and Oakeshott [5]. However, the same basic picture has a longer pedigree in the rock mechanics literature, and can be traced at least as far back as the pioneering work of Trollope in the 1950's [17]. (The latter is very clearly reviewed in [18].) Something very like the Edwards-Oakeshott model is called by Trollope the "full arching limit" and something very like the BCC model turns out to be the "no arching limit" of Trollope's theory (although his predictions 
based on the latter do not take proper account of the Coulomb yield criterion, as was done by BCC). Trollope also developed a "systematic arching theory" to provide an interpolation between these two limits. This model, though it does not provide a systematic theory of arching, is quite interesting, and we discuss it in more detail in Section 2.10. It is based on quite different physical principles from our own work, partly because Trollope attributed the arching phenomenon to small displacements of the supporting surface under a wedge. This idea, based on Trollope's own experiments (on wedges whose construction history we have been unable to find out) is clearly at odds with our own explanation, and appears to be contradicted by the more recent experimental data on sandpiles constructed from a point source [1, 2, 19].

\subsection{Related modelling work}

Other approaches to the problem of stress propagation in static sandpiles include particle packing models [3, 4], where one considers a regular packing of (usually spherical) grains, with simple transmission laws for the downward force between one layer of particles and those below. These models show a flat stress plateau in two dimensions, a feature shared with BCC [7] which can be viewed as a (slightly generalized) continuum limit of such models. An important and related class of discrete models address the propagation of noise effects in sandpiles; in these the transmission of forces between particles is stochastic [20, 21]. The relation between these models and our own (noise-free) continuum approach will be explored in detail elsewhere [22].

More elaborate discrete models are increasingly being studied. That examined numerically by Bagster and Kirk [8] invokes nontrivial force propagation rules locally, and for some parameter ranges shows a dip in the stress. However, it is not clear whether the physics included in this model is that of real sandpiles; and so far, the relation to any continuum description is uncertain. A widespread numerical approach is that of discrete element modelling [23]; however, as discussed by Buchholtz and Poeschel [24], many such methods cannot so far even reproduce the fact that the repose angle of a pile is independent of its size. Various improved algorithms have been suggested [25]; we do not know whether the same is true for these. In a future paper 26] we will present results from a simulation approach involving nominally frictionless, but nonspherical, slightly deformable particles, following Ref.[24]. These offer some promise of confirming, or at least testing, the ideas put forward in the present work.

The model of Liffman et al. [9] invokes a more specific mechanism, based on sizesegregation, to explain the dip in the stress. When confronted with the experimental data [1, 2] one sees a serious drawback of this explanation: the data show a scaling behaviour which the model cannot support. The observed scaling (called RSF scaling, see Sec. 2.3 below) indicates that there is no characteristic length-scale intrinsic to the granular medium of which a pile is made. In general, segregation effects introduce such a scale by setting up gradients in the material properties of the pile [27], and hence violate the observed scaling. It is notable that a finite deformability of the particles would also introduce a characteristic length, and is therefore also ruled out by RSF scaling (we discuss this further in Sec. 2.3).

The IFE model, defined above, represents one limiting case of a more general group of elasto-plastic continuum theories; some of these are highly developed and widely used within a finite element framework (though usually in the context of hoppers rather than 
sandpiles) 28\|. The physical basis of these models for dry cohesionless granular media is not always clear (many are based on models developed earlier for wet soil [13]). In any case, to whatever extent elasticity is invoked, such models are again in violation of RSF scaling.

The idea that the properties of a granular medium depend on its construction history is central to our work. This concept is not new, and plays a strong role in the recent experimental literature on granular media in hoppers (for example the exit flow from a hopper depends on how it was filled) [29]. Indeed, this is part of the reason why standardized shear and triaxial tests are used to measure the internal friction coefficient of a granular medium; the repose angle, which in the simplest theories is completely equivalent [10], in reality depends appreciably on construction history, as do other mechanical properties [29] (this is discussed further in Section 4). Moreover, it has long been argued that the manner in which the construction history enters is via the local packing geometry of the grains. This forms part of the idea of "granular fabric", in which one constructs a local tensor that parameterizes the distribution of particle-particle contacts [30. The concept of the fabric tensor has usually been developed in an elasticity context, rather than one in which constitutive relations directly among stresses are assumed. Nonetheless, the orientational memory effects embodied in the new models described below can certainly be viewed in terms of a local tensorial property of the medium (see Sec. 2.7). However, no specific interpretation of this quantity (in terms of the contact distribution) appears to be required.

\subsection{The present work}

In Section 2 we give a coarse-grained continuum description of the two-dimensional symmetrical sandpile. Instead of assuming in advance a particular constitutive relation, we first approach the problem systematically by exploiting the implications of symmetry, and of the boundary condition of incipient failure at the free surface (IFS). We discuss, with reference to the construction history of the pile, the scaling ansatz of a radial stress field (RSF). This ansatz transforms the partial differential equations into ordinary differential equations, which can be solved easily for all the closures considered later. Using this, and the idea of perfect memory mentioned above, the range of possible constitutive relations is greatly reduced.

After this, we will solve our equations for four specific closures in two dimensions; these are incipient failure everywhere (IFE), Bouchaud-Cates-Claudin (BCC), fixed principal axes (FPA), and finally the family of oriented stress-linearity (OSL) closures, which includes BCC and FPA as special cases. All of these comply with our modelling strategy of seeking local constitutive relations among stress components. For the OSL model, stresses propagate along straight characteristics which can be interpreted by analogy with wave propagation along "light-rays". We thereby arrive at a very simple geometrical picture of stress propagation, from which the forms of the stress profiles can be swiftly deduced. For the OSL model, the effect of a small perturbation (adding a little extra weight somewhere) is studied, and an appropriate Green function described. This helps sharpen the idea of stress being carried along arches.

In Section 3 we extend our calculations to the three dimensional conical sandpile. Because of the larger number of stress components (some of which can be eliminated by symmetry) a second constitutive equation is now required. We study several possible 
choices for this secondary closure, and find that all of these lead to qualitatively similar stress profiles. A comparison with the experimental results of Smid and Novosad [2] is then made. This shows, firstly, that the RSF scaling assumption is well-verified, and, secondly, that the data is fit rather well by the FPA model, without adjustable parameters. Viewed alternatively as a comparison with the OSL model, which does have an adjustable parameter (the tilt angle), the evidence suggests that parameter values close to the FPA case must be chosen. The data thereby presents strong evidence for the arching picture as an explanation of the dip.

Until the end of Section 3, we will have considered only the case where the free surface of the pile is at the angle of repose. However, it is possible experimentally to achieve piles which are flatter than this. We discuss this and a number of related problems in Section 4, where, for simplicity, we restrict attention to the FPA model in two dimensions. We show that it matters how a sandpile is made: for example, if a flattened pile is created by slicing wedges off the top of a steeper one, the stresses should differ from those found by choosing a material with a lower repose angle to begin with. It is in geometries such as this, that the dependence of the constitutive equation on the construction history of the pile can be probed.

Section 5 contains a brief summary of our approach and a concluding discussion. Our calculations for the stress propagation in two-dimensional sandpiles, using the OSL and FPA models, are new; as are our three dimensional results for these models, and for BCC, although some of the FPA results were outlined elsewhere [31]. For the IFE model, which is more classical, the corresponding results may exist in the literature (though we have not found them); in any case we include them for comparison.

\section{The two dimensional symmetrical sandpile}

\subsection{Indeterminacy of stress continuity equation}

As mentioned previously, the continuum approach to calculating the stress distribution in a static sandpile immediately encounters an indeterminacy. Indeed, the stress continuity equation in two dimensions reads (componentwise)

$$
\begin{aligned}
& \partial_{r} \sigma_{r r}+\partial_{z} \sigma_{r z}=0 \\
& \partial_{r} \sigma_{r z}+\partial_{z} \sigma_{z z}=g
\end{aligned}
$$

which, clearly, provides only two relations between the three independent elements of the stress tensor $\sigma_{z z}, \sigma_{r r}$ and $\sigma_{r z}=\sigma_{z r}$.

To ease the later generalization to three dimensions (Section 3) we here use cylindrical polar coordinates, with $z$ measured downward from the apex of the pile and $r$ a radial coordinate from the symmetry axis - see Fig.1(c). (In two dimensions, $r=|x|$, with $x$ a cartesian coordinate.) We have assumed that the granular medium has constant density, $\rho$, thereby excluding segregation effects (see Section 1.2 above), and have chosen units where $\rho=1$. The acceleration due to gravity is denoted $g$; because it enters linearly, this could also be set to unity, but we retain it for clarity. The stress tensor $\sigma_{i j}$ is defined to be symmetric in $i, j$, as usual in the physics literature.

In our cylindrical polar coordinate system the stress tensor is a function of $z$ and $r$, where $r \geq 0$ by definition. However, in terms of cartesians $(z, x)$, one would have 
both positive and negative $x$; in this case, the normal stresses would be even functions of $x$ and the shear stress an odd function [7]. (The latter holds because the unit vector along $r$ reverses sign at the symmetry axis.) Confusion can be reduced by restricting attention to the left half of the pile (positive $x$ ) for which the two coordinate systems coincide. In any case, on the symmetry axis itself $(r=0)$, the shear stress must vanish by symmetry, and the $z$ and $r$ directions are both principal axes there.

As well as the three stress components $\sigma_{r r}, \sigma_{z z}$ and $\sigma_{z r}$, it will be useful to consider the following three quantities: the average stress $P=\left(\sigma_{r r}+\sigma_{z z}\right) / 2$, the "radius of Mohr's circle" $R$ defined via $R^{2}=\left(\sigma_{z z}-\sigma_{r r}\right)^{2} / 4+\sigma_{z r}^{2}$ [32], and the (positive) angle of inclination $\Psi$ between the $z$-axis to the major principal axis of the stress tensor (see Fig.1(c)). In terms of these,

$$
\begin{aligned}
\sigma_{r r} & =P-R \cos (2 \Psi) \\
\sigma_{z z} & =P+R \cos (2 \Psi) \\
\sigma_{z r} & =R \sin (2 \Psi)
\end{aligned}
$$

Following the usage of the engineering literature [10] we define a material point to be in an active state if the normal stress $\sigma_{z z}$ in the direction of the external compressive force (here gravity) is larger that the stress $\sigma_{r r}$ perpendicular to it. On the symmetry axis $(r=0)$, the $z$-axis is the major principle axis and $\Psi=0$ in the case of an active state, whereas for a passive state the major axis is $r$, and $\Psi= \pm \pi / 2$.

The simplest model of a granular medium is known as the ideal cohesionless Coulomb material. The Coulomb model plays the same role in the study of granular materials as the Newtonian fluid does in viscous flow, and we will use it here. Plastic failure occurs in a given material element if there exist a plane defined by a unit normal $\mathbf{n}$ (or angle of inclination $\left.\tau=\sin ^{-1}(\mathbf{n} . \hat{\mathbf{z}})\right)$ through this element, on which the shear forces $\sigma_{n m}$ exceeds a given fraction of the normal force $\sigma_{n n}$ across the plane [33]. Conversely the element is stable if, for all such planes,

$$
\left|\sigma_{n m}\right| \leq \tan (\phi) \sigma_{n n}
$$

For a material with cohesion, a constant $c$ is added to the right hand side; we treat only the cohesionless case. The Coulomb yield criterion can then alternatively be expressed as

$$
\Upsilon \equiv \frac{R}{P \sin (\phi)} \leq 1
$$

(Put differently, "the yield locus must not cut Mohr's circle" [32].) The coefficient $\tan (\phi)$ in eqn.(3) is the coefficient of static friction of the material; elementary arguments show that $\phi$ is then the angle of repose [10] (defined, as usual, as the inclination of the free surface to the horizontal; see Fig.1(c)).

\subsection{IFS boundary conditions}

We now use the yield criterion to specify the stresses on the surface of the pile. In doing this, we neglect the small Bagnold hysteresis angle (as mentioned in Sec. 1.1) and demand that the surface of a pile, constructed from a point source and at its angle of repose, is in a state of incipient slip. (Sandpiles constructed differently, for which this is not the case, are considered in Section 4). 
First we note that (in two dimensions) all stress components have to vanish on the surface:

$$
\sigma_{r r}(S=1)=\sigma_{z z}(S=1)=\sigma_{z r}(S=1)=0
$$

Here we have introduced, for reasons that will be clarified later, a scaling variable $S=r /(c z)$ with $c=\cot (\phi)$. (Hence the equation of the free surface is $r=c z$, or $S=1$.) The vanishing of the stresses is a direct consequence of the yield criterion, as we now show by considering the stress components in a rotated coordinate system $(n, m)$ (see Fig.1(c)). For a system inclined at angle $\tau$ to the vertical, one has

$$
\begin{aligned}
\sigma_{n n} & =\cos ^{2}(\tau) \sigma_{r r}+\sin ^{2}(\tau) \sigma_{z z}-2 \sin (\tau) \cos (\tau) \sigma_{z r} \\
\sigma_{m m} & =\sin ^{2}(\tau) \sigma_{r r}+\cos ^{2}(\tau) \sigma_{z z}+2 \sin (\tau) \cos (\tau) \sigma_{z r} \\
\sigma_{n m} & =-\sin (\tau) \cos (\tau)\left(\sigma_{z z}-\sigma_{r r}\right)+\left(\cos ^{2}(\tau)-\sin ^{2}(\tau)\right) \sigma_{z r}
\end{aligned}
$$

Now choosing $\tau=\pi / 2-\phi$, so that $n$ is normal to the surface, we require that the normal stress $\sigma_{n n}$ at the free surface has to vanish (this assumes that no external forces act there). The yield criterion eqn. (4) then requires

$$
\sigma_{m m}^{2} \cos ^{2}(\phi)+4 \sigma_{n m}^{2} \leq 0
$$

Accordingly, the remaining two stress components $\sigma_{m m}$ and $\sigma_{n m}$ must also vanish, and the stress tensor is zero in the $(n, m)$, and hence in the $(r, z)$, coordinate system.

The criterion that the surface of the pile is a slip plane, not only implies that the stresses on the surface vanish, but also fixes their ratios in its immediate neighbourhood. Demanding equality in eqn. (3) as the surface is approached, we obtain the condition

$$
\lim _{S \rightarrow 1} \frac{\sigma_{n m}(S)}{\sigma_{n n}(S)}=-\tan (\phi)
$$

(the sign can be confirmed from eqn. (6)). Applying also eqn.(7) (with equality) in this limit gives a second condition:

$$
\lim _{S \rightarrow 1} \frac{\sigma_{m m}(S)}{\sigma_{n n}(S)}=1+2 \tan ^{2}(\phi) \equiv 1 / \eta_{0}
$$

where the final notation will prove convenient later. By rotating using eqn. (6) these can be written in the $(r, z)$-system as

$$
\begin{aligned}
\lim _{S \rightarrow 1} \frac{\sigma_{z r}(S)}{\sigma_{r r}(S)} & =\tan (\phi) \\
\lim _{S \rightarrow 1} \frac{\sigma_{r r}(S)}{\sigma_{z z}(S)} & =\eta_{0}
\end{aligned}
$$

(The results in $(m, n)$ and in $(r, z)$ coordinates look rather similar because, as it turns out, the two frames are related by a reflection through the major principal axis [34].)

The requirements expressed by equations (5, 10), represent a set of "boundary conditions" which we denote IFS (incipient failure at the surface). Along with the stress continuity equation, these form the boundary value problem for determining the stress profile of a sandpile. At first sight there may appear to be more boundary conditions than required; however (as emphasised before) to close the problem in two 
dimensions, we will need a constitutive relation between stress components, which is yet to be chosen. One can therefore view any extra "boundary conditions" as constraints limiting this choice. We next develop some general scaling arguments which, combined with some other physically motivated simplifications, further restrict the choice of constitutive equation.

\section{$2.3 \quad$ Scaling analysis}

The basis of our scaling approach is to assume that the macroscopic material properties of a granular medium (under gravity) are independent of length scale. Obviously, any such medium has a characteristic length associated with the grain size, but in a continuum description this should not be important. The scaling hypothesis supposes that not only this length, but also any other characteristic length-scale that the medium possesses, is either extremely small, or else extremely large, compared to the scales probed in a macroscopic sandpile experiment. As mentioned previously, and shown below, our scaling assumption is well-verified in the experiments of Ref. [2]. However, those experiments are on piles of a limited size range (20 to $60 \mathrm{~cm} \mathrm{high}$ ). It is possible that for smaller or larger piles our scaling assumptions would break down, due to a characteristic length arising from size segregation [9], for example. A corollary of our scaling assumption is that no relevant intrinsic scale exists for stresses: otherwise, this scale could be compared with the gravitational stress to give a length. Thus we exclude, for example, deformable particles whose elastic modulus sets a stress scale, and thereby a "deformation length" (which for rigid particles is infinite). These simpifications, though guided by experiment, are not physically obvious a priori; the problem deserves more careful experimental study to determine the limits to the scaling regime for real granular materials.

Assuming that the medium indeed has no intrinsic characteristic length, the stress distributions in all piles formed the same way (of the same material) should be similar. Hence we search for a scaling solution of eqn. (11) of the form:

$$
\sigma_{i j}=g z s_{i j}(S)
$$

with the scaling variable $S=r / c z$ was introduced previously. In anticipation of this ansatz, our earlier discussion of the boundary conditions was couched in terms of $S$. These boundary conditions impose $s_{i j}(1)=0$, and also fix ratios of derivatives of $s_{i j}$ (see eqns.(5.10) above). The functions $s_{i j}$ have to be continuous everywhere; however, we are dealing with hyperbolic equations, and the stresses need not be differentiable [35.

The scaling ansatz, eqn. (11), reduces the partial differential equations for stress continuity, eqns. (1), to the following ordinary differential equations:

$$
\begin{aligned}
& s_{r r}{ }^{\prime} / c+s_{z r}-S s_{z r}{ }^{\prime}=0 \\
& s_{z r}{ }^{\prime} / c+s_{z z}-S s_{z z}{ }^{\prime}=1
\end{aligned}
$$

The primes denote derivatives with respect to the scaling variable $S$; recall that $c=$ $\cot (\phi)$. Solutions of eqn. (12) are usually called "radial stress fields" [36] and we refer to (11) as RSF scaling. ¿From the scaling behaviour of $s_{i j}$ follows a corresponding scaling 
of the mean stress, $P(r, z)=g z \tilde{P}(S)$, the radius of Mohr's circle, $R(r, z)=g z \tilde{R}(S)$, and the angle of the major principle axis $\Psi(r, z)=\Psi(S)$.

We now ask, what are reasonable closure relations consistent with the radial stress field form of eqn. (11)? On physical grounds we first impose the requirement of locality: the unknown stress component in a material element depends on the known ones in that element, and not elsewhere. (As mentioned previously, this would fail if distant loads were able to rearrange the grains themselves in a given neighbourhood.) The most general form consistent with our scaling ansatz is then

$$
\sigma_{r r} / \sigma_{z z}=C\left(\sigma_{z r} / \sigma_{z z}, S\right)
$$

where the absence of an intrinsic stress scale, noted above, means that only dimensionless ratios of stresses can enter.

To restrict $C$ further, we now invoke the assumption of perfect memory mentioned in the introduction: that the constitutive relation in a material element is determined at the time of its burial and is not subsequently altered. Now, it is clear that each material element is buried while just at the surface of the pile $(S=1)$, after which it experiences continually decreasing values of $S$ as the pile gets larger. Hence any explicit dependence of $C$ on $S$ would violate the perfect memory assumption. Accordingly, we must have

$$
\sigma_{r r} / \sigma_{z z}=C(U)
$$

where we have defined the reduced shear stress

$$
U(S)=\sigma_{z r} / \sigma_{z z}=s_{z r} / s_{z z}
$$

An exception to the above argument should be made for material elements on the central axis, which are buried, and remain forever, with a value $S=0$. In two dimensions the centreline divides grains which have rolled to the left from those which have rolled to the right, and which therefore have had qualitatively different histories [37. Accordingly, there is no requirement that $C$ behave smoothly at the origin; and although the constitutive models studied below all appear analytic when expressed in polar $(z, r)$ coordinates, for some of them $C$ does become singular on the symmetry axis, when a cartesian $(z, x)$ coordinate system is employed. (The models with this property are the OSL models, including FPA, but with the exception of BCC.)

We can now reformulate the radial stress field eqns. (12) as

$$
\begin{aligned}
\frac{d s_{z z}}{d S} & =\frac{\hat{c} \hat{e}-\hat{b} \hat{f}}{\hat{b} \hat{d}-\hat{a} \hat{e}} \\
\frac{d s_{z r}}{d S} & =\frac{\hat{a} \hat{f}-\hat{c} \hat{d}}{\hat{b} \hat{d}-\hat{a} \hat{e}}
\end{aligned}
$$

Here we have introduced the notations

$$
\begin{aligned}
& \hat{a}=-\frac{1}{c} \frac{U^{2} d(C(U) / U)}{d U} \\
& \hat{b}=-S+\frac{1}{c} \frac{d C(U)}{d U} \\
& \hat{c}=s_{z r}(S)
\end{aligned}
$$




$$
\begin{aligned}
& \hat{d}=-S \\
& \hat{e}=1 / c \\
& \hat{f}=s_{z z}(S)-1
\end{aligned}
$$

where only the first two functions explicitly involve the closure relation. Similarly we can also reformulate the Coulomb yield criterion, eqn. (代) as:

$$
1 \geq \Upsilon(U)^{2}=\frac{(1-C(U))^{2}+4 U^{2}}{(1+C(U))^{2} \sin ^{2}(\phi)}
$$

eqns. (16) give a systematic procedure for solving (at least numerically) the boundary problem for any specified constitutive relation $C$; eqn. (18) then allows one to check its stability. The latter step is necessary to ensure that the yield criterion (marginally satisfied at the surface) is not violated deep inside the pile; any closure for which such violations arise must be rejected.

\subsection{Asymptotic behaviours}

Without specifying $C$ further, we can now use the scaled continuity equations, eqn. (12), to examine the possible asymptotic behaviours close to the free surface, and close to the symmetry axis of the pile.

As mentioned previously, all the stresses vanish at the free surface $(S \rightarrow 1)$. Asymptotically we expect them to vanish as power laws $s_{r r}=a_{1}(1-S)^{\alpha}, s_{z z}=b_{1}(1-S)^{\beta}$, $s_{z r}=d_{1}(1-S)^{\delta}\left(a_{1}, b_{1}, d_{1}, \alpha, \beta, \delta>0\right)$. The IFS condition, eqn. (10), requires that $s_{r r}$ and $s_{z z}$ have to vanish with the same power, $\alpha=\beta$, since their ratio approaches a constant. More generally one finds by substituting the power law forms into eqn. (12) that the stresses on the surface have to vanish linearly : $\alpha=\beta=\delta=1$. This applies for any choice of the closure $C$. (For the models solved below, this linear behaviour is visible in Fig.2(a).) Only one of the coefficients $a_{1}, b_{1}, d_{1}$ then remains free; using (12) we find $d_{1}=a_{1} / c$ and $-d_{1} / c+b_{1}=1$. Using again the IFS boundary condition, eqn. (10), we obtain finally 38

$$
\begin{aligned}
a_{1} & =1 /\left(1+\tan ^{2}(\phi)\right) \\
b_{1} & =\left(1+2 \tan ^{2}(\phi)\right) /\left(1+\tan ^{2}(\phi)\right) \\
d_{1} & =\tan (\phi) /\left(1+\tan ^{2}(\phi)\right)
\end{aligned}
$$

This completes the specification of the asymptotic behaviour near the free surface. The average reduced stress on the surface is $\tilde{P}=1-S$ and the reduced Mohr's radius $\tilde{R}=\sin (\phi)(1-S)$. Since, from eqn. (2) $) \cot (2 \Psi)=\left|\left(b_{1}-a_{1}\right) / 2 d_{1}\right|$ we can solve for the direction of the major principal axis at the free surface

$$
\Psi(1)=\psi \equiv(\pi-2 \phi) / 4
$$

This asymptote for $\Psi(S \rightarrow 1)$ bisects the angle between the vertical and the free surface itself.

A similar analysis can be made of the stresses close to the symmetry axis of the pile $(S \rightarrow 0)$. Again without knowing details of $C$, we can establish for $S \rightarrow 0$ a solution involving a linearly vanishing shear stress $s_{z r}=d_{0} S$, a vertical normal stress of the 
form $s_{z z}=b_{0}+b_{c u s p} S$, and a flat horizontal normal stress $s_{r r}=\eta b_{0}$. Here $\eta \equiv C(0)$ is the ratio of horizontal and vertical stresses in the middle of the pile. One can also show that $b_{0}=1-d_{0} / c$, with $b_{0}, d_{0}$ positive. The existence of a cusp, $b_{\text {cusp }} \neq 0$, is associated with a breakdown in the smoothness of the closure relation $C$ on the symmetry axis; as discussed above, this is physically permissible, and is a distinguishing feature of the new models introduced in this paper.

The results of this and the preceding section were obtained by combining RSF scaling with the perfect memory assumption, without further restriction on $C$. In the next few sections we finally consider various model constitutive relations with which to close the equations and thereby calculate explicit stress profiles for the sandpile.

\subsection{The IFE model}

The traditional [12, 10, 11] assumption of Incipient Failure Everywhere (IFE) means that the granular material is everywhere marginally unstable; the frictional forces are fully mobilized and $\Upsilon=1$. (Accordingly the two principal stresses are everywhere in fixed ratio.) Indeed, we can solve eqn. (18) with equality to find:

$$
C(U)=\frac{1}{\cos ^{2}(\phi)}\left(\left(\sin ^{2}(\phi)+1\right) \pm 2 \sin (\phi) \sqrt{1-(\cot (\phi) U)^{2}}\right)
$$

where $U(S)=s_{z r} / s_{z z}$ is the reduced shear stress introduced previously. Here the negative sign must be chosen: this corresponds to requiring downward (rather than upward!) incipient slip of the grains at the free surface. The resulting IFE constitutive equation then fixes the two functions $\hat{a}(S), \hat{b}(S)$ defined earlier, as follows:

$$
\begin{aligned}
\hat{a} & =\frac{1}{\cos ^{2}(\phi)}\left(1+\sin ^{2}(\phi)-2 \sin (\phi) \sqrt{1-(\cot (\phi) U)^{2}}\right) \\
\hat{b} & =-s+\frac{2 U}{c \sin (\phi) \sqrt{1-(\cot (\phi) U)^{2}}}
\end{aligned}
$$

thereby closing the the radial stress field equations (16). We have not obtained an analytical solution for this model, but a numerical solution was readily found by a standard Runge-Kutta procedure (by shooting from the middle of the pile to the boundary conditions on the surface). The reduced stresses $s_{z z}, s_{r r}$ and $s_{z r}$ are shown for the case $\phi=30^{\circ}$ in Fig.2(a). (Also shown are results from the other models discussed below.) The IFE model give a smooth "hump" in $s_{z z}$.

\subsection{The BCC model}

In place of the IFE assumption that the principal stresses are proportional, the BCC model [7] assumes instead the proportionality of vertical and horizontal normal stress components:

$$
\frac{\sigma_{r r}}{\sigma_{z z}} \equiv C(U)=\eta
$$

This assumption, which is perhaps the simplest possible, is related (but not identical) to one made in the classical work of Janssen [39, 10, 12]. Invoking also the IFS boundary conditions, complete results were obtained analytically for the two dimensional sandpile 
[7]. These results for the stresses, the yield function $\Upsilon$ and the orientation angle $\Psi$ of the major principal axis are shown (alongside those of IFE and FPA) in Figs.2(a-c).

The IFS condition (eqs. (0, 8, 9) in fact requires $\eta=\eta_{0}$ (where $\eta_{0}$ is defined in eqn.9). The stresses are piecewise linear with a cusp at $S=S_{0}=c_{0} / c$, where $c_{0}=\sqrt{\eta}_{0}$ and, as shown by BCC, $c_{0} / c$ is strictly less than unity. The inequalities $c_{0} / c \leq S \leq 1$ define an "outer region" of the pile in which the stresses obey $s_{z z}=(1-S) /\left(1-\left(c_{0} / c\right)^{2}\right)$ and $s_{z r}=(1-S) c_{0}{ }^{2} c /\left(c^{2}-c_{0}^{2}\right)$. These match at $S=S_{0}$ onto an "inner region" $(0 \leq S \leq$ $\left.c_{0} / c\right)$, in which there is a plateau for the vertical normal stress, $s_{z z}=1 /\left(1+c_{0} / c\right)$, while the shear stress vanishes linearly on the central axis, $s_{z r}=S c_{0} /\left(1+c_{0} / c\right)$. As shown in eqn.(20) above, the inclination angle of the major axis obeys at the surface $\Psi(1)=\psi$. For the BCC closure in two dimensions, this value is maintained throughout the outer region, while in the inner region $\Psi(S)$ obeys $\tan (2 \Psi)=2 c_{0} S /\left(c_{0}^{2}-1\right)$. Hence $\Psi(S)$ vanishes smoothly at $S=0$.

\subsection{The Fixed Principal Axis (FPA) model}

Neither the IFE nor the BCC closure gives a "dip" in two dimensions (nor in three as shown below). We therefore propose a new hypothesis 31 which appears to capture, within a fully consistent continuum theory, the physics of arching (as expounded by Edwards and coworkers [5, 6]). Specifically, we postulate that the major principal axis of the stress tensor has a fixed angle of inclination to the downward vertical: $\Psi(S)$ is constant. We first describe the results and afterwards discuss in more detail the physical content of this model.

The FPA hypothesis provides a local constitutive equation by assuming that the principal stress axes in a material element have constant orientation fixed at the time of its burial. However, with the exception of those lying right on the symmetry axis, all such elements were first buried at the surface of the pile $(S=1)$. Since the IFS boundary condition already fixes $\Psi(1)=\psi \equiv(\pi-2 \phi) / 4$, our FPA model requires

$$
\Psi=\psi
$$

everywhere. Using the results of Sec. 2.1, one finds immediately that this is equivalent to the following constitutive relation:

$$
\frac{\sigma_{r r}}{\sigma_{z z}} \equiv C(U)=1-2 \tan (\phi) U
$$

Note that if $r$ is replaced by $x$ (cartesian coordinates) this becomes

$$
\frac{s_{x x}}{\sigma_{z z}} \equiv C(U)=1-2 \operatorname{sign}(x) \tan (\phi) U
$$

The $\operatorname{sign}(x)$ factor is a reminder that, in the FPA model (unlike BCC) our $C(U)$ is nonanalytic at the symmetry axis of the pile. A compact way to write eqn. (26]) is [31] $C(U)=1-2 \tan (\phi)|U|$, though (see Section 4 below), this version is not equivalent for all construction histories.

Since the repose angle $\phi$ (and thereby $\psi$ ) is a material parameter fixed by experiment, the FPA model gives a complete closure of the $d=2$ sandpile problem. The resulting equations are linear. Their structure is clearest when written in terms of the 
unscaled stress components $\sigma_{i j}$; substituting eqn.(25) and eqn.(15) into eqn.(11) (taking $\sigma_{z z}$ and $\sigma_{r z}$ as the independent variables) gives:

$$
\begin{aligned}
\partial_{r} \sigma_{r z}+\partial_{z} \sigma_{z z} & =g \\
\partial_{r}\left(\sigma_{z z}-2 \tan \phi \sigma_{r z}\right)+\partial_{z} \sigma_{r z} & =0
\end{aligned}
$$

which can be rewritten

$$
\left(\partial_{z}-c_{1} \partial_{r}\right)\left(\partial_{z}-c_{2} \partial_{r}\right) \sigma_{i j}=0
$$

with

$$
\begin{aligned}
c_{1}+c_{2} & =-2 \tan (\phi) \\
c_{1} c_{2} & =-1
\end{aligned}
$$

where we $c_{1}\left(c_{2}\right)$ to denote the positive (negative) roots. A little manipulation then shows that $c_{1}=\tan (\psi)$ and $c_{2}=-\cot (\psi)$. A similar equation for the stresses, but with $c_{1}=-c_{2}=\sqrt{\eta_{0}}$, was obtained for the BCC model [7], in which case eqn.(29) becomes the wave equation in two dimensions. We shall call (29) a "wave equation" even when $c_{1}+c_{2} \neq 0$; under these conditions, it becomes an ordinary wave equation (with equal velocities) if tilted coordinate axes are chosen 40.

A complete solution is readily found and is given explicitly (in the context of the more general OSL model) in the next Section. As with BCC, one finds for $s_{z z}(S)$ a piecewise linear function, with inner and outer regions. The material in the outer region again saturates the yield criterion (18) whereas the inner part does not; these regions are separated by a cusp at $S=S_{0}=c_{1} / c$. For the FPA model, there is always a dip in $s_{z z}$ at the centre of the pile. The dip takes the form of a cusp at $S=0$ and is connected with the nonanalyticity of $C(U)$, which reflects the sudden change in the direction of the major principal axis on passing through the central axis of the pile. The maximum vertical stress (at $\left.S=S_{0}\right)$ is a factor $\left(1+2 \tan ^{2}(\phi)\right)$ times larger than the value at $S=0$ (the latter is always finite). These results are compared with the $\mathrm{BCC}$ and IFE models in Fig.2(a-c).

At first sight the requirement of fixed orientation of the stress tensor $(\Psi=\psi)$ is at odds with the fact that, on the centre line of the pile, there are no shear stresses and so the horizontal and vertical directions must be principal axes $(\Psi=0)$. This paradox can be resolved by noting that on the centre line the stress in the FPA model is actually isotropic, thus satisfying both criteria at once.

The correspondence between the FPA model and the Edwards arching picture becomes clearer on considering the characteristics of the wave equation, (29), which are straight lines of slope $c_{1}$ and $c_{2}$ (as discussed further in Section 2.9 below), Fig.3. Since for the FPA model $c_{1}=\tan (\psi)$ and $c_{2}=-\cot (\psi)$, the characteristics are at rightangles to one another; moreover, they coincide with the principal axes of the stress tensor. It is this special property of the FPA model that we believe embodies Edwards' physical picture of arches [5, 6]. The stress arising from the weight of an element of sand propagates along two straight characteristics, one at $\psi$ to the vertical (which we identify as the "arch direction", coincident with the major principal axis) and the other at rightangles. As shown in Section 2.9 below, the majority of the stress is carried by the outward characteristic (slope $c_{1}$ ). The material can therefore be viewed as a set of nested arches (Fig.1(b)) down which most of the stress propagates. (This ties in with 
Edwards' idea of "lines of force" [6].) However, a minority of the stress is transmitted instead from one arch to its inner neighbour; this transfer imparts mechanical stability to the outer, incomplete, arches. Since the principal axes and the characteristics coincide, there are no shear forces acting at the interface between successive arches, which are therefore effectively in frictionless contact with one another. This seems to be as close as one can get, within a consistent continuum theory, to the intuitive picture of arches as independent load-bearing structures.

We emphasize that for a sandpile constructed from a point source, the FPA model can be viewed in two ways; either as a macroscopic hypothesis concerning the transmission of stresses at the scale of a pile (principal axes fixed in space), or as a microscopic hypothesis concerning the way the growth history of the pile is locally encoded. Our own modelling approach, based on local constitutive relations among stresses, corresponds to the latter view. Indeed, after making the assumption of perfect memory, we have to choose one scale-free property (the constitutive equation) to be "remembered" by any element from the moment of its burial: and the choice made by FPA corresponds to remembering the orientation of the stress tensor (principal axes fixed in time). Note that, once the basic FPA assumption is made, there is no free parameter left in the theory (at least, not in two dimensions), since $\phi$ is fixed by experiment.

It seem plausible that the construction of the pile (by a series of avalanches at the surface) imparts to the local packing of grains a permanent sense of direction. If so, the FPA constitutive relation is perhaps the simplest model for how this "orientiational memory" within an element could determine the constitutive relation among stresses arising there subsequently. A possible (though not a necessary) interpretation of this directional memory is in terms of a fabric tensor $\lambda_{i j}$ [30]. For example, one could postulate that $\lambda_{i j}$ was constant throughout the medium (when expressed in cylindrical polar coordinates) and moreover had a principal axis $\Psi$ bisecting the free surface and the vertical. If this were true, the FPA constitutive relation would reduce to the commutation requirement $\sigma_{i j} \lambda_{j k}=\lambda_{i j} \sigma_{j k}$.

Apart from its appealing simplicity, however, we have no detailed mechanistic justification for the FPA model in terms of the fabric tensor or any similar quantity: why should the orientation of the principal axes be remembered, rather than something else? (For example, in the IFE model each element "remembers" instead that it was at the critical threshold for slip when buried, and remains so forever after.) We therefore propose the FPA model as a phenomenological hypothesis to be tested against experiment. It is interesting, in that context, to consider alternative closures; we do this next, by embedding the FPA model within a broader scheme.

\subsection{The Oriented Stress Linearity (OSL) model}

A sandpile is formed by layerwise deposition of particles that have rolled down its free surface. Thus the grains of sand may end up arranged in a packing that locally distinguishes the directions toward and away from the central axis. An arching effect can arise if this anisotropy tends to direct stresses outward from the centre, thus "screening" the central part of the pile from the added load of new layers. This offers a possible way to explain the dip; and indeed the FPA model can be viewed in exactly these terms. However, it is not unique in this respect.

A more general approach can be generated by an adaptation of the $\mathrm{BCC}$ model, 
in which it was assumed that $\sigma_{r r}=\eta \sigma_{z z}$. BCC thus singles out for special treatment the vertical and horizontal normal stresses. We now define the oriented stress linearity (OSL) model by assuming a similar linear relationship between normal stresses, not in a $(z, r)$ coordinate system, but in a tilted one $(n, m)$. The latter system is now characterized by an arbitrary (but constant) tilt angle $\tau$ to the vertical, and is related to $(z, r)$ via the transformation equation (6). (In general this $n, m$ system does not coincide with the one used earlier to discuss the IFS boundary condition.) In the tilted coordinates, we now require (following BCC) that the two normal stresses, $\sigma_{n n}$ and $\sigma_{m m}$ are proportional:

$$
\sigma_{n n}=K \sigma_{m m}
$$

(see Fig.1(c)). Despite its formal similarity, the OSL closure differs critically from $\mathrm{BCC}$ in that it violates the assumption, tacitly made by $\mathrm{BCC}$, that the properties of the medium vary smoothly as one passes through the centre line of the pile; it thereby allows a cusp (dip or hump) to arise in $s_{z z}$.

Leaving the angle $\tau$ and the constant $K$ free for the moment, we use the rotation eqn. (6) to obtain, in $(z, r)$ coordinates, the OSL constitutive relation

$$
\frac{\sigma_{r r}}{\sigma_{z z}}=C(U)=\eta+\mu U
$$

(where, as always, $U=\sigma_{r z} / \sigma_{z z}$ ). As with FPA, in cartesians $(z, x)$ this becomes

$$
\frac{\sigma_{x x}}{\sigma_{z z}}=C(U)=\eta+\mu \operatorname{sign}(x) U
$$

whereby the singularity on the centreline becomes apparent.

The constants $\eta$ and $\mu$ obey:

$$
\begin{aligned}
\eta & =\frac{K-\tan ^{2}(\tau)}{1-K \tan ^{2}(\tau)} \\
\mu & =\frac{2(K+1) \tan (\tau)}{1-K \tan ^{2}(\tau)}
\end{aligned}
$$

Clearly the BCC model corresponds to $\eta=\eta_{0}(\phi)$ (defined in eqn.9) and $\mu=0$, whereas the FPA model, eqn. (25), is obtained by setting $\eta=1, \mu=-2 \tan (\phi)$. Both are thereby special cases of OSL [41]. Note that pairs of OSL coordinate systems inclined through angles $\tau$ and $\tau+\pi / 2$ (or $\tau-\pi / 2$ ) are identical, subject to interchanging the $m$ and $n$ axes; they give the same values of $\eta$ and $\mu$ in (33) and hence the same stress profiles in the pile.

The coefficients $\eta$ and $\mu$ (or equivalently $K$ and $\tau$ ) in the OSL model are not independent: an equation between them can be found from the IFS boundary condition as formulated in eqns. (10). For a given repose angle $\phi$, this condition restricts the OSL parameters to the "IFS line" :

$$
\eta=\eta_{0}(1-\mu \tan (\phi))
$$

Hence the OSL model has one remaining free parameter (unlike BCC or FPA, which have none). The IFS lines in the $(\mu, \eta)$-plane are shown for two values of the friction angle $\phi$ in Fig.4. 
The OSL constitutive relation eqn.(33) can be substituted into the stress continuity equations (11) to give

$$
\begin{aligned}
\partial_{r} \sigma_{r z}+\partial_{z} \sigma_{z z} & =g \\
\partial_{r}\left(\eta \sigma_{z z}+\mu \sigma_{r z}\right)+\partial_{z} \sigma_{r z} & =0
\end{aligned}
$$

from which we can obtain a wave equation of the form (29), as discussed already in the context of the FPA model. In this more general case, however, $c_{1}$ and $c_{2}$ are the positive and negative roots respectively of

$$
c_{1,2}=\frac{1}{2}\left(\mu \pm \sqrt{\mu^{2}+4 \eta}\right)
$$

The propagation velocities become equal in magnitude if coordinate axes are rotated by the tilt angle $\tau$.

The resulting stress propagation equations can be solved without difficulty. As with the FPA model, there are inner and outer regions which meet at $S=S_{0}=c_{1} / c$; in the outer region we obtain

$$
\begin{aligned}
s_{z z} & =s_{*}(c-\mu)(1-S) \\
s_{r r} & =s_{*} \eta c(1-S) \\
s_{z r} & =s_{*} \eta(1-S)
\end{aligned}
$$

where we have introduced the constant

$$
s_{*}=\frac{c}{c^{2}-\mu c-\eta}=\frac{c c_{1}}{\left(c c_{1}+\eta\right)\left(c-c_{1}\right)}
$$

In the inner region $\left(0 \leq S \leq c_{1} / c\right)$ we find

$$
\begin{aligned}
s_{z z} & =s_{*}\left(c-c_{1}\right) / c\left(c_{1}-\mu S\right) \\
s_{r r} & =s_{*} \eta c_{1}\left(c-c_{1}\right) / c \\
s_{z r} & =s_{*} \eta\left(c-c_{1}\right) / c S
\end{aligned}
$$

As stated already for FPA and BCC, we thus obtain stress profiles that are piecewise linear functions of $S$. We see from (41) that a dip in $s_{z z}$ is present so long as $\mu<0$. This applies for OSL models on the part of the IFS line which lies in the left hand half plane, Fig.4; such models are separated by the BCC model $(\mu=0)$ from OSL models with a hump $(\mu>0)$.

As described earlier in connection with eqn. (18), a further check on the consistency of the model should now in general be made: we require that the yield threshold is not exceeded within the pile. The above equations show that the threshold is exactly saturated, not only at the surface (IFS) but throughout the outer region. However, there is also the possibility of yield in the inner region; when this happens, it first occurs at the very centre of the pile [42]. In this neighbourhood, where shear stresses are negligible, the Coulomb criterion eqn. (18) simplifies to

$$
\begin{array}{r}
\eta_{\min } \leq \eta \leq \eta_{\max } \\
\eta_{\min }=\frac{1-\sin \phi}{1+\sin \phi}=\eta_{\max }^{-1}
\end{array}
$$


Where $\eta_{\min }$ is known as "Rankine's coefficient of active earth pressure" [10]. Thus, for a given repose angle $\phi$, acceptable OSL parameters lie on the the segment of the IFS line bounded by eqn.(42) (dash-dotted lines in Fig.4). Outside this range, there is either too deep a dip or too high a hump, leading respectively to passive or active failure of the material at the centre of the pile.

The FPA model lies in the $(\eta, \mu)$ plane at the point where the IFS line crosses $\eta=1$. It divides those OSL models which, on the central axis of the pile, have active behaviour $(\Psi(0)=0)$, from those which are passive there $(\Psi(0)= \pm \pi / 2)$. For the OSL models generally, the orientation of the principal axes varies smoothly as one passes from left to right through the centre of the pile (though the constitutive equation is nonanalytic there). The sole exception to this is FPA, which has instead a discontinuity in $\Psi$ at $S=0$, for the reasons discussed in the previous section. This highlights the fact that FPA is the only model in the OSL family for which the geometrical picture of "nested arches" (Fig.1(b) and Sec. 2.6 above) can definitely be said to apply.

\subsection{Linear models and "light-rays"}

We have seen that in the OSL model, the stresses propagate with a wave equation in which $c_{1}$ and $c_{2}$ are the positive and negative roots of eqn.(38). The characteristics of this hyperbolic equation are thus straight lines of slopes $c_{1}$ and $c_{2}$. This means that, if a perturbation is made at some point in the pile (for example, increasing the weight of a certain element of sand), the resulting information travels along two "light rays" (together called a "light cone" in Ref.[7]). Since the stress propagation equations are linear, the entire stress distribution can be constructed by summing the contributions from all elements of sand propatated along suitable rays; this offers an instructive geometric insight into the problem.

First we consider the Green function which describes the stress perturbation arising from a point source of weight. Such a source term violates the left-right ("cylindrical") symmetry of our two-dimensional system; to deal with it we must introduce cartesians $(z, x)$ as opposed to the polar coordinates $(z, r)$ used so far. In such coordinates, eqn. (29) is virtually unchanged:

$$
\left(\partial_{z} \pm c_{1} \partial_{x}\right)\left(\partial_{z} \pm c_{2} \partial_{x}\right) \sigma_{i j}=0
$$

where the + signs apply for $x>0$ and - for $x<0$. Our source term then consists of adding $\Delta g(z, x)=\delta\left(x-x_{0}\right) \delta\left(z-z_{0}\right)$ to the right hand side of the second member of the stress continuity equation (11), in which $x$ now replaces $r$. This yields an inhomogeneous form of (43) with derivatives of the delta-function on the right hand side. The algebraic form of the Green function is complicated (we do not write it out explicitly here) but its geometric interpretation is relatively simple, as shown in Fig.5(a).

Of the stress $\sigma_{z z}$ contributed by a small element of sand, a fraction $A_{1}$ propagates along the outward light ray and $A_{2}$ along the inner ray. (Note that $A_{1}+A_{2}=1$ : the vertical normal stress is a conserved quantity in $z$.) A ray of amplitude $A_{i}$, with velocity $c_{i}$, also carries shear and horizontal normal stresses $\sigma_{x z}=c_{i} \sigma_{z z}$ and $\sigma_{x x}=c_{i}^{2} \sigma_{z z}$. (Here $i=1,2$; these relations may be confirmed by direct application of the wave equations). Because shear stress is also conserved in $z$ (for a point source of weight, the $x$-integral of $\sigma_{x z}$ is zero), one has $A_{1} / A_{2}=\left|c_{2} / c_{1}\right|$. 
Since the wave velocities $c_{1}$ and $c_{2}$ become reversed as one crosses the centreline, in all cases (except for the BCC model where $c_{1}=-c_{2}$ ), this line forms a boundary between two different wave media and any ray impinging on it undergoes both reflection and refraction. For simplicity we now move the origin of our $z, x$ coordinates to the point where the ray meets the centreline, in which case an incident ray emanating from our point source corresponds to a disturbance $\sigma_{z z}=A_{2} \delta\left(x-c_{2} z\right)$, whereas the reflected ray obeys $\sigma_{z z}=R A_{2} \delta\left(x-c_{1} z\right)$, and the transmitted ray $\sigma_{z z}=T A_{2} \delta\left(x+c_{1} z\right)$ (this incorporates the sign change of $c_{1}$ on crossing the centreline). The factors $R$ and $T$ can be deduced as follows. First, one imposes the conservation law for $\sigma_{z z}$ defined above; the total weight supported by the reflected and transmitted rays is the same as that in the incident ray. This yields immediately $R+T=1$. Secondly, by considering the force on a small element, one finds that not only the shear stress but also the horizontal normal stress $\sigma_{x x}$ must be continuous across the centreline. (Note that the same does not apply, in general, to the vertical normal stress.) Imposing this for the normal stresses, we equate $\sigma_{x x}\left(x=0^{+}\right)=A_{2} c_{2}^{2} \delta\left(x-c_{2} z\right)+A_{2} R c_{1}^{2} \delta\left(x-c_{1} z\right)$ with $\sigma_{x x}\left(x=0^{-}\right)=A_{2} T c_{1}^{2} \delta\left(x+c_{1} z\right)$. Using also the fact that $\delta(x-c z)=|c|^{-1} \delta(x / c-z)$, we find a second relation, $\left|c_{1} / c_{2}\right|+R=T$. Thus we obtain the results

$$
\begin{aligned}
& T=\left(\left|c_{2} / c_{1}\right|+1\right) / 2 \\
& R=\left(1-\left|c_{2} / c_{1}\right|\right) / 2
\end{aligned}
$$

which completes our analysis of the reflection/refraction processes. Note that for OSL models with dip $(\mu<0)$ the reflected ray factor $R$ has to be negative.

The above argument shows that the stress response at height $z$ to a point source above this level in the pile consists of either two delta functions (amplitudes $A_{1}$ and $A_{2}$ ) or three delta-functions (amplitudes $A_{1}, A_{2} R$ and $A_{2} T$ ) according to whether or not the inward-going ray from the source has met the centre-line. Using this information we can construct a geometrical solution of the wave equations for each stress component; for the vertical normal stress $\sigma_{z z}(x)$ at a point $x$ on the base (say), this is done as follows (Fig.5(b)). From each of two points separated by a small distance $\Delta x$ centred on $x$, construct the backward light rays (allowing for any reflection at $x=0$ ). This defines two strips of material, one of length $L_{1}$ and the other of length $L_{2}$, with a third and fourth each of length $L_{3}$ if there is a reflected/refracted ray. The corresponding widths $w_{1}, w_{2}$ (and $w_{3}=w_{4}$ ) are as shown in the figure. The total vertical normal force between our two points now obeys

$$
\sigma_{z z}(x) \Delta x=g\left(A_{1}\left(w_{1} L_{1}+R w_{3} L_{3}\right)+A_{2}\left(w_{2} L_{2}+T w_{3} L_{3}\right)\right)
$$

In other words, one adds the stress contributions of all the material elements which have a light ray ending in the given interval $\Delta x$ on the base. The above construction provides a formula for $\sigma_{z z}$ which is, of course, identical to eqns.(39,41) derived earlier. Since the other stress components also obey a wave equation, each of these can be constructed similarly, as a weighted sum of the three L's. The fact that each stress component is a piecewise linear function of $x$ then follows from the elementary geometry of triangles.

The Green function construction shown in Fig.5 gives some direct insight into the role of the arching concept in describing stress propagation in granular media. The relation between the characteristic slopes $c_{1,2}$ and the arching effect is far from intuitive, 
however. Specifically we can ask how, starting from the BCC model $\left(c_{1}+c_{2}=0\right)$ one should adjust the tilt parameter $(\tau)$ of the OSL model so as to obtain an arching effect, and thereby a dip in the stress. In a slightly different language [16], this was addressed briefly in Ref. [7], where it was suggested that to get an arching behaviour the light rays emanating from an element would have to be tilted outwards $\left(\tau>0\right.$, or $\left.c_{1}+c_{2}>0\right)$, thus transporting the load away from the centre. This suggestion, though at first sight reasonable enough, is actually wrong. In the FPA model, and all other OSL models giving a dip, the light rays are actually tilted inward relative to the BCC model: their average slope, $\left(c_{1}+c_{2}\right) / 2$ is negative $(\tau<0)$. This would, at first sight, appear to carry the weight of the grains toward the centre of the pile. The paradox is resolved by realizing that, on tilting the rays inwards, the amplitudes $A_{1}, A_{2}$ defined above, adjust so that $A_{1}$ becomes larger than $A_{2}$. This means that a higher fraction of the weight of a grain is carried along the outward ray, and away from the centre of the pile; this redistribution is more than enough to compensate for the average inward tilt of the two rays.

\subsection{Trollope's Model}

As mentioned in the Introduction, Trollope 17, 18 proposed a model which yields, in effect, Edwards' arches and BCC as its two limiting cases. The relation between this model and our own work is most clearly seen in terms of the above analysis using rays; we therefore discuss it now.

In his model, Trollope, without invoking any differential formulation of the problem, directly assumed that the stress could be constructed in a manner similar to that above, but using three rays; two with equal and opposite velocities $c_{1}=-c_{2}$ (just as in the BCC model), and a third, horizontal ray $\left(c_{3}=-\infty\right)$. The parameter $c_{1}$ was taken as fixed globally by the type of packing; however, a second parameter $k$ was introduced. This $k$ represents an imposed amplitude ratio $A_{2} / A_{1}=k$ for the outward and inward components of the BCC-like propagation; as $k$ is varied, the amplitude $A_{3}$ of the third ray also changes (in a manner that can be deduced from stress continuity). It is interesting that Trollope already realized the importance of singular behaviour on the centreline; for $k \neq 1$, his model has this property.

In the limit $k=1$ (no arching, symmetric propagation), $A_{3}$ vanishes and one recovers a BCC-like picture (though unlike BCC, Trollope did not connect $c_{1}$ to the repose angle). In the limit $k=0$ one again has two rays, one of which is now horizontal. This limit does not correspond to any OSL model however: within the OSL model an infinite $c_{2}$ (representing the horizontal ray) automatically has zero amplitude: $A_{2}=0$. Trollope's horizontal ray enables stress continuity to be satisfied while giving a maximal dip (zero normal stress $\sigma_{z z}$ at $x=0$ ), reminiscent of the Edwards approach. (The Coulomb yield criterion is violated, however.)

By use of the third ray, Trollope managed to interpolate these limits in what he called the "systematic arching theory". However, the introduction of this extra ray seems extremely ad-hoc, which is perhaps why the model is not more widely used today. Mathematically its presence means that rays can no longer be identified as characteristics of a partial differential equation in two dimensions; therefore Trollope's construction cannot correspond to any local constitutive equation among stresses. (All such closures must lead to hyperbolic equations for which a formal solution using 
characteristics is available, even if the characteristics are curved - as happens, for example, in the IFE model [10].) We conclude that Trollope's systematic arching theory must be rejected as unphysical - a view tacitly shared by most of the recent sandpile literature. However, many of the physical ideas behind the model, including the emphasis on discontinuities in propagation across the centreline, remain highly pertinent to the present work.

\section{The conical sandpile}

We now extend our continuum modelling approach to the three dimensional conical sandpile.

\subsection{One additional missing constitutive equation}

The conical pile is as shown in Fig.1(d); in addition to the cylindrical coordinates $(z, r)$ introduced before, an azimuthal coordinate $\chi$ is required. Since we have axial symmetry around the $z$-axis, the principal axes of the stress tensor must include the azimuthal $(\chi \chi)$ direction. (The orientation of this tensor can thus be fully specified, as before, by the inclination angle $\Psi$ to the vertical of the major principal axis in the $r, z$ plane.) Recalling that the stress tensor is symmetric, we therefore have $\sigma_{r \chi}=\sigma_{\chi_{r}}=\sigma_{z \chi}=$ $\sigma_{\chi z}=0$. Hence the three dimensional conical pile has only one additional independent stress component $\sigma_{\chi \chi}$ compared to the two dimensional case [7]. The stress continuity equation for a conical sandpile is

$$
\begin{aligned}
\partial_{r} \sigma_{r r}+\partial_{z} \sigma_{r z} & =\frac{\sigma_{\chi \chi}-\sigma_{r r}}{r} \\
\partial_{r} \sigma_{r z}+\partial_{z} \sigma_{z z} & =g-\frac{\sigma_{z r}}{r} \\
\partial_{\chi} \sigma_{i j} & =0
\end{aligned}
$$

The first two equations differ from those found earlier in two dimensions by additional "source terms", $\left(\sigma_{\chi \chi}-\sigma_{r r} / r\right)$ and $-\sigma_{z r} / r$ respectively, on the right hand side.

Because of the high symmetry of the conical pile, closure of these equations requires only that we find two constitutive equations which together should determine any two of the independent stress components in terms of the remaining two. Choosing the latter as before $\left(\sigma_{r z}\right.$ and $\left.\sigma_{z z}\right)$ we refer to the resulting equation for $\sigma_{r r}$ as the primary, and that for $\sigma_{\chi \chi}$ as the secondary constitutive equation. Note that symmetry requires also

$$
\begin{aligned}
\sigma_{z r}(r=0) & =0 \\
\sigma_{r r}(r=0) & =\sigma_{\chi \chi}(r=0)
\end{aligned}
$$

As our yield criterion for plastic failure of the granular material we retain the Coulomb criterion 44] for cohesionless granular materials (which becomes a relation between principal stresses in the $r, z$ plane). However, the Coulomb yield criterion is essentially two-dimensional in character and gives no explicit information on the circumferential stress $\sigma_{\chi \chi}$. In common with previous authors 44 we argue nonetheless that this should vanish on the free surface, as all the other stress components do:

$$
s_{\chi \chi}(S=1)=0 .
$$


Note that if we were to use instead the Conical Yield criterion 110, 13, 43] or a similar (fully three dimensional) condition at the surface, eqn. (48) would not be an extra assumption.

The form of the new source terms in eqn. (46) is of interest. If these remain relatively small everywhere, one can expect to find (independent of the form of chosen for the secondary closure relation) qualitatively similar results to those obtained earlier in the two dimensional case. This scenario is indeed fulfilled for the various different secondary closures tried below. In any case, given that all stresses vanish at the surface (as just described), these source terms become strictly negligible near the free surface of the pile, which may therefore be viewed locally as having a planar two-dimensional geometry. Accordingly, the IFS boundary condition is completely unaffected; the stresses on the surface of a pile obeying IFS are still given by eqns.(8,9, 10). It follows that (subject to the usual scaling assumptions, see below) eqns. (19) and (20) still govern the asymptotic behaviour near the free surface. Thus the relation between the repose angle $\phi$ and parameters in the primary constitutive equation (such as the tilt angle $\Psi$ in the FPA model, or the $\eta$ and $\mu$ parameters in OSL) remain as they were in two dimensions.

\subsection{Scaling analysis}

As for the two dimensional case, by invoking the absence of an intrinsic length scale we may demand that solutions of of the stress continuity equation take the RSF scaling form, eqn. (11). Substituting this into eqn. (46) gives a set of ordinary differential equations:

$$
\begin{aligned}
s_{r r}{ }^{\prime} / c+s_{r r}+s_{z r}-s_{\chi \chi}-s s_{z r}{ }^{\prime} & =0 \\
s_{z r}{ }^{\prime} / c+s_{z r}+s_{z z}-s s_{z z}{ }^{\prime} & =1
\end{aligned}
$$

The asymptotic analysis in Section 2.4 for the stresses near the surface carries over to the case of three dimensions, as mentioned already above. The source terms in eqn. (46) can in principle affect the the asymptotic behaviour given in Section 2.4 near the centre of the pile (i.e., small $S$ ) but qualitative changes arise only if either $\sigma_{z r} / r$ or $\left(\sigma_{r r}-\sigma_{\chi \chi}\right) / r$ become large in this limit. This does not occur for any of the models studied below.

Following the arguments made earlier in two dimensions, based on our assumptions of RSF scaling and "perfect memory", we now propose local forms for both the primary and secondary constitutive relations, which must be as follows:

$$
\begin{aligned}
& s_{r r} / s_{z z}=C(U) \\
& s_{\chi \chi} / s_{z z}=D(U)
\end{aligned}
$$

where we have set $U=s_{z r} / s_{z z}$ as usual.

The form of eqn. (16) for the RSF scaling solution remains basically unchanged, except that in eqn. (17) the terms $\hat{c}=s_{z r}+s_{r r}-s_{\chi \chi}$ and $\hat{f}=s_{z z}+s_{r z}-1$ are somewhat modified. The resulting stress profiles can readily be calculated numerically from eqns. (16,17) for any choice of the closure relations. However, no analytic solution appears to be obtainable even for those models which, in two dimensions, reduce to wavelike propagation. The problem can, of course, still be viewed as quasi twodimesional (one spacelike, one timelike variable), but if so the extra "source terms" 
make the solution complicated. Alternatively these models can be formulated in terms of wave propagation in two spacelike dimensions (the $r, \chi$ plane) with $z$ as a timelike variable. However, the Green function for such waves is itself surprisingly complicated (there is no sharp "light-cone" [7]) and not directly amenable to the simple geometrical interpretations offered earlier.

\subsection{Choice of constitutive equations}

For the primary constitutive equation, we can choose among those discussed earlier, namely IFE and OSL, with the latter including both BCC and FPA as special cases. We continue to require that the IFS boundary condition is obeyed at the surface (which again fixes the OSL parameters $\mu$ and $\eta$ to lie on the IFS line) and that the Coulomb yield criterion is not violated in the interior of the pile.

For the secondary constitutive equation, we have investigated three ways of selecting the function $D(U)$. The first is to insist that $D(U)$ coincides with $C(U)$ so that $\sigma_{\chi \chi}=\sigma_{r r}$ everywhere in the pile:

$$
D_{1}(U) \equiv C(U)
$$

This has the merit of simplicity. (Note that by symmetry this relation must hold anyway at the central axis of the pile, but not necessarily elsewhere.) A second choice is suggested by the observation that the $\chi \chi$ direction is a minor principle axis for a conical sandpile with axial symmetry. Generalizing slightly an assumption often made the context of conical hoppers [13, 10], one could then choose as the secondary closure $\sigma_{\chi \chi}=P-R$ (the Haar - von Karman hypothesis [13). This implies

$$
D_{2}(U)=(1+C(U)) / 2-\sqrt{(1-C(U))^{2} / 4+U^{2}}
$$

Although the motivation for this choice in the sandpile context is not very clear, we have tried it out for comparison. Our third choice of secondary closure, unlike the first two, does not explicitly depend involve the primary closure $C(U)$; it is the linear relation

$$
D_{3}(U)=\eta+\tilde{\mu} U
$$

which should be compared with the OSL primary closure, eqn.(33). In fact, for the OSL model the constant term $\eta$ has to be identical to that chosen in the primary closure, to meet the second requirement of eqn. (47). The coefficient $\tilde{\mu}$ is in principle free. In practice, however, we have found that the requirement that the Coulomb criterion $\Upsilon \leq 1$ holds in the interior of the pile means that values of $\tilde{\mu}$ close to $\mu$ are required; hence for OSL models the closure $D_{3}(U)$ is never very different from $D_{1}(U)$.

We have investigated these three closure relations $D_{1}, D_{2}, D_{3}$ for all the different primary closures $C$ already discussed in Section 2, for various values of the repose angle $\phi$ (mainly in a range around $\phi=30^{\circ}$ ). For all the parameters we tried, the extra "source terms" led mainly to smoothing of the two dimensional curves without qualitatively altering the presence or absence of the dip. Since these source terms do not have a dramatic effect, it follows that the choice made for $D$, at least among those investigated here, itself does not qualitatively change the stress profiles. 


\subsection{Results}

Rather than provide a catalogue of curves for various combinations of primary and secondary closure, we will focus attention on the FPA model. In Fig.6 we compare the stress curves for the three-dimensional FPA with closures $D_{1}$ and $D_{2}$. As mentioned previously, the choice of secondary closure proves quantitatively but not qualitatively important. Also shown are the experimental results of Ref. [2] for piles of height 20$60 \mathrm{~cm}$. The stresses are normalized by the total weight of the pile; notice the good scaling collapse of curves from piles of varying heights. This confirms that the RSF scaling hypothesis made in this paper is obeyed to experimental accuracy, at least for the materials and pile sizes studied in Ref. [2]. The agreement between experiment and FPA theory is generally satisfactory, although there is a significant error near the maximum of the vertical normal stress. Obviously it would be helpful to have more data for small values of $S$, but there are sufficient data points at the origin to clearly establish the presence and magnitude of the dip. The experimental data shown are for two different media both with repose angles close to $\phi=33^{\circ}$. The resulting curves differ by an amount similar to the difference between the two choices of secondary closure, with $D_{1}$ giving slightly better results for "quartz sand" and $D_{2}$ for "NPK-1 fertilizer". (We do not attach any significance to this.) As mentioned previously, the FPA model has no adjustable parameters once $D$ is chosen and $\phi$ is fixed by experiment.

In Fig. 7 we show the same predictions for the FPA model with closure $D_{1}$ alongside those for several other models with the same secondary closure. These models are BCC and IFE (neither showing a dip); and two parameter choices for the OSL model $(\eta=0.8$ and $\eta=1.2)$ which bracket the FPA case $(\eta=1)$. This comparison shows a clear preference of FPA over those other models that have no adjustable parameter. It is conceivable that the data could be fit better by choosing an OSL model with $\eta$ slightly different from unity. However, we do not believe the improvement is enough to justify the adoption of an extra fitting parameter, although further careful experiments might reveal this to be necessary.

Finally in Fig.8 we plot the yield parameter $\Upsilon(S)$ for the BCC, FPA and IFE models. By definition, $\Upsilon(S)=1$ everywhere in the IFE model; it also obeys $\Upsilon(1)=1$ in all models obeying the IFS boundary condition. In two dimensions it is also unity throughout the outer regime of the pile for all OSL models. In three dimensions this is not the case, and in fact for OSL models the material is clearly below the yield criterion throughout the bulk of the pile. This underlies the important distinction between the classical IFE assumption (fully mobilized friction, $\Upsilon=1$ ) and the new models adopted in this paper.

\section{Role of construction history}

So far we have only considered the stress profiles of idealized sandpiles constructed by pouring sand from a stationary pipe, for which the boundary condition of incipient failure at the surface (IFS) was assumed. The slope $\alpha$ to the horizontal of the free surface is by definition given by the repose angle: $\alpha=\phi$. According to our approach, however, the constitutive equation encodes the construction history, and piles built differently can behave differently. In discussing this issue, we restrict attention to the FPA constitutive model in two dimensions. 
We consider first the following hypothetical experiment: a material with $\phi=\phi_{0}$ is formed into a pile by the usual method. The pile is then reduced to a flatter (symmetrical) one of angle $\alpha$ by simply taking away the upper section, grain by grain, without disturbing any material below (Fig.9(a)). According to our model, the constitutive equation remains that of a pile with the larger repose angle, though the stresses are of course altered. The resulting stress pattern is shown in Fig.9(b) in comparison to that of a pile of repose angle $\phi_{1}=\alpha$ which has the same final geometry. The first of the two piles has the larger dip. We can now ask the following: if a pile of $\alpha<\phi$ is tilted from the base through an angle $t$ (Fig.9(c)) how large may $t$ become before an avalanche occurs? A classical answer, based on the view that the repose angle $\phi$ is a material property independent of construction history, is that one would be able to tilt until $t_{\max }+\alpha$ is again equal to $\phi$. (This ignores, as we have done throughout this paper, the small hysteresis effects associated with the Bagnold angle [14]). However, in our approach this should not quite be true, since the inclination angle of the principal axes in a pile at this condition is different (by an angle $t$ ) from that of a pile created by the normal method at its repose angle. It turns out, however (Fig.9(d)) that unless the pile is substantially flattened $\left(\phi-\alpha \simeq 10^{\circ}\right.$ or more), the difference between $t_{\max }+\alpha$ and $\phi$ is very slight, at least for repose angles in the usual range $\left(\phi<45^{\circ}\right)$.

This calculation can, with caution, be proposed as a model for what happens when a sandpile, built normally, is suddenly tilted through a finite angle $t$. Of course, in this case an avalanche does occur: however, if this happens by removal of a wedge without significant reorganization of the remaining part of the pile, leaving the new surface in a state of incipient failure, the above calculation can be applied (except that, for simplicity, we have contrived a version in which the pile remains symmetrical). In principal, there should then be a change in the resulting repose angle if $t$ is large enough. However, the assumption that an avalanche occurs with no rearrangement of the remaining grains, is, for large $t$, highly dubious.

Another critical test of our ideas is the following: a triangular pile is constructed as usual and then a large part of it removed (grain by grain) leaving a pile whose left hand slope is at the angle of repose $\phi$, and whose right hand slope is at angle $\beta$ (say) to the horizontal. The geometry is chosen so that all of the material in the new pile was originally in the left half of the parent pile (Fig.10(a)). To describe this situation, we have to use the FPA constitutive equation in the form (26), in a coordinate system where $x=0$ denotes the centreline of the original pile. With the modified construction history just described, the singularity on the line $x=0$ lies outside the newly created pile, and the characteristics of stress propagation should be identical on both sides of its apex. Throughout the pile, a majority of the stress is carried down the leftmost (rather than outermost) characteristic. An interesting question now is, what is the maximum angle $\beta$ that we can choose for the right hand slope? This can be found from the usual stability criterion $\Upsilon \leq 1$; the marginal case has equality at the free surface on the right and, for the FPA model, this gives (after some algebra) the condition

$$
\tan (\psi-\beta) \geq \tan ^{3}(\psi)
$$

The maximum $\beta$ for which the new pile is stable is shown as a function of $\phi$ in Fig.10(b); for $\phi=30^{\circ}$, one has $\beta_{\max }=19.1^{\circ}$. This is a very interesting result, since it predicts that the repose angle of the right hand part of a pile built this way is quite different from the usual value, which prevails on the left. 
This prediction must, of course, be interpreted with caution since its extension to a fully three dimensional geometry is not obvious. Perhaps the simplest three dimensional analogue is to build a pile and then open a hole directly below the vertex, allowing sand to flow out leaving a "volcano crater" 445]; according to this prediction, the angle of repose on the inner side of the crater may differ substantially from that on the outer slope. This possibility deserves careful experimental study; a significant difference is not ruled out [46]. The situation is again complicated by the fact that the experiment will set up a flow which may rearrange the grains that remain in the pile. Indeed, the removal by avalanche of the right hand part of the pile may set up a large region in which the grains have slipped down to the right, for which the constitutive equation may revert to that of the right hand part of a normal pile. (This could be true even if the actual particle displacements are extremely small.) If so, the measured repose angle could again approach $\phi$, rather than $\beta_{\text {max }}$ which applies only when the removal of sand does not perturb the remainder. We show in Fig.10(c) the stress distribution in a pile made in this careful fashion (with $\phi=30^{\circ}$ and $\beta=15^{\circ}$ ). As one might expect, there is now no dip but a (lopsided) maximum in the vertical normal stress. The maximum lies to the left of the new apex (at the point where an outgoing ray from this apex strikes the base).

Note that quite different predictions for this geometry could have been obtained by writing the FPA closure in a somewhat different form, which is, for a symmetrical pile only equivalent to eqn. (26) 31], as discussed in Section 2.7:

$$
s_{x x} / s_{z z}=1-2 \tan \phi|U|
$$

Here the explicit dependence on construction history via the $\operatorname{sign}(x)$ factor has been replaced by an $x$-independent but highly nonlinear constitutive relation among the stresses (in the spirit of some of the models discussed in Ref.[[]]). Using this form, one could find a solution, with $\beta=\phi$, for our asymmetrically constructed pile that would precisely coincide with the usual symmetrical case. (This possibility arises because the sign of $U$, though not of $x$, can change on the centreline of the new pile.) Since we only have data for symmetric piles, we cannot on the existing facts rule out this rather different version of the FPA model, although it does not correspond to our assumption that the principal axes of a material element are fixed at the time of burial. Accordingly experiments on asymmetric piles would be a strong test of the theory.

A somewhat different experiment would be to start with a symmetrical pile and then remove parts of it (grain by grain) so that the remainder forms an asymmetrical pile whose apex has not moved from the line $x=0$. Shown in Fig.11 is the stress distribution in such a pile with left and right slopes $\alpha_{1}=\phi=30^{\circ}$ and $\alpha_{2}=12^{\circ}$. An interesting feature is visible under the apex, where the vertical normal stress $\sigma_{z z}$ (and therefore also the yield function $\Upsilon$ ) is discontinuous. This behaviour is in fact a generic feature of sandpiles that include the line $x=0$ but are asymmetric about it. It stems from the fact that this normal stress is not continuous in the geometry of incident, reflected and transmitted rays considered earlier (Section 2.9). An exception to this rule is if an asymmetrical pile is made by pouring sand onto a sloping base plate. In this case, we expect the apex of the pile to move slightly relative to the source so that more material rolls down the "long" side of the pile and the repose angle in the two directions remain equal to $\phi$. The constitutive equation must then (given RSF scaling) be the same as for a pile formed normally, and the stress exerted on the supporting 
plate is the same as that on an inclined plane inscribed through a normal pile. (In two dimensions, this can be found easily from our earlier results.) Though asymmetric, this stress distribution will not show any discontinuity beneath the apex.

As a last example of a sandpile constructed normally and then manipulated, in Fig.12 we show the stress distribution in a pile whose top section has simply been cut off. Though grain-by-grain removal of sections of a pile may impose experimental difficulties, this is perhaps the simplest geometry for which it could be achieved. As shown, the dip is gradually diminished and replaced by a plateau as larger and larger upper sections are taken away.

Finally, we note that a sandpile could be made by first distributing sand uniformly (not from a point source) in a retaining bin, from which the side walls are then removed. As with some of the examples studied above, the predictions depend crucially on whether significant slip occurs within the part of the pile that finally remains. The initial loading of the bin is likely to produce principal axes with vertical and horizontal orientations $(\Psi=0)$, so that if no slip occurs, we would expect the BCC model to apply (no dip). However, if slip does occur so the remaining pile has been sheared downwards, the FPA picture should be more appropriate.

The various types of experiment discussed above, in which the construction history of the pile is deliberately manipulated, provide a strong test of our basic modelling hypothesis that the constitutive equation encodes the construction history. For some of these geometries, the theoretical predictions challenge the "classical" assumption, maintained in the recent physics literature on sandpiles, that for cohesionless granular media (of a single grain size [10]) the repose angle is the same for all types of pile of a given material. (As mentioned in Sec. 1.2, this assumption has long been avoided in the engineering literature on hoppers [46, 29].) Of course, the repose angle remains a genuine material parameter in that the angle of a "normal" pile (built from a point source) will differ for different cohesionless materials; and for our purposes this can be taken as the unique definition of $\phi$. It does not necessarily follow, however, that the repose angle taken up by a pile of the same material with a different construction history, will always be exactly the same. In any case, our modelling approach leads to a clear expectation that the stresses in such piles can be different (even when the repose angles are not significantly different). This is a readily testable prediction which we believe deserves urgent experimental attention.

\section{Conclusion}

This paper is a long one. It therefore seems useful to provide a brief summary of our modelling strategy, in the form of a list of contentions for which more detailed arguments can be found in the text above. We stress, however, that several points on the list have no first-principles justification: they are hypotheses whose value can at present only be judged by comparison with experiment. We also stress that several of these ideas have a long history (which is not the same as saying that they are widely agreed upon). 


\subsection{A manifesto for sandpile modelling}

Our modelling strategy is based on the following claims:

(1) There is a construction history, $\mathcal{H}$. This determines the arrangement of grains. We define the "normal" history to be the construction from a point source of a pile at its repose angle.

(2) There is a stress tensor $\sigma_{i j}$ which is well-defined as a local (mesoscopic) average over many grains.

(3) For hard particles (of infinite elastic modulus), no strain variables exist; static frictional forces are indeterminate. Stress continuity requires one supplementary equation for closure in two dimensions, and two for a conical pile in three dimensions.

(4) Scaling behaviour (RSF scaling) is observed, to experimental accuracy. Hence there is no characteristic length scale in a sandpile under gravity. Particle deformability would provide such a length; so would size segregation.

(5) The limit of uniform nondeformable, cohesionless, particles presumably therefore exists, and should describe those experiments for which RSF scaling is observed.

(6) We should therefore seek as closure a scale-free, local constitutive relation among stresses. Formally: there is a function $\mathcal{C}$ such that

$$
\mathcal{C}\left(\sigma_{i j}(r, z), \mathcal{H}\right)=0
$$

The constitutive relation depends on the local packing and therefore on the construction history: $\mathcal{C}$ encodes $\mathcal{H}$.

$(7) \mathcal{C}$ for a material element is "frozen in" at the time of burial (perfect memory assumption). Combined with RSF scaling, this means that for a sandpile constructed from a point source, $\mathcal{C}$ is independent of position when expressed in cylindrical polar coordinates, though it may be singular on the central axis.

(8) The boundary conditions for a pile constructed normally are IFS: incipient failure at the free surface. This means that at the surface, the major principal stress axis bisects the free surface and the downward vertical. (Here and elsewhere, hysteresis effects associated with the Bagnold angle are ignored.)

(9) The search for a constitutive relation $\mathcal{C}\left(\sigma_{i j}, \mathcal{H}\right)$ may legitimately entail (a) making simplified hypotheses to compare with experiment; (b) microphysical modelling from first principles. We pursue the former in this paper, the latter elsewhere [26].

(10) A classical choice of $\mathcal{C}$ is incipient failure everywhere (IFE); this is hard to defend physically. It does not predict a dip in the stress beneath the apex of a pile.

(11) A physically more plausible (but by no means unique) choice for $\mathcal{C}$ is provided by the FPA hypothesis. According to this, each element of material is impressed at burial with a sense of direction, which fixes forever the orientation of the stress tensor ellipsoid that the element can support. The model predicts a dip in two dimensions.

(12) In three dimensions, a secondary closure relation is needed. Among the more obvious choices, it makes relatively little difference which is chosen. Even the simplest choice $\left(\sigma_{\chi \chi}=\sigma_{r r}\right)$, combined with FPA, gives a reasonably good fit to the data of Ref.[2], without adjustable parameters.

(13) A generalized model (OSL), of which FPA is a special case, can be introduced. This has an adjustable parameter, the introduction of which is not demanded by the present data. 
(14) The above modelling approach, though initially set up for static sandpiles constructed from a point source, can also be used for more complex construction histories (at least in some cases). For piles constructed normally and then modified by careful removal of grains, this approach predicts a nontrivial dependence of the repose angle $\phi$, and of the stress distribution, on the way a pile is made.

\subsection{Discussion}

Of the models considered in this paper, it is clear that the FPA model has some especially attractive features. This model leads directly to an arch-like stress-propagation, with the major part of any load being carried down the arch direction. The latter coincides with the major principal axis of the stress tensor; this everywhere bisects the free surface and the downward vertical. The predictions of the FPA constitutive relation thereby describe similar physics to the arching model of Edwards [5] (and indeed the earlier "full arching theory" of Trollope [18]). Like such models, the FPA hypothesis can be viewed as a direct macroscopic ansatz of how stresses propagate: one assumes that the principal axes are fixed in space. Viewed this way, we believe that the FPA model provides the first description of the arching picture within a fully consistent continuum mechanics framework. Its experimental success strongly suggests that the presence of a macroscopic arching structure in sandpiles is the correct explanation for the observed minimum in the vertical normal stress below the apex of the pile.

However, unlike previous arching models, the FPA hypothesis can also be interpreted as providing a local, history-dependent constitutive relation among stresses. In this context, it is among the simplest such equations that can plausibly be devised: we assume that the principal axes of a material element are fixed at the time of its burial. Viewed as such, the FPA hypothesis contains no assumption of any macroscopic arching structure; rather, it provides a plausible microscopic explanation for how such structures arise. Its experimental success offers strong support for a modelling strategy cast in terms of such constitutive relations. For parameter values other than FPA, which is a special case, the more general OSL model predicts, within the same modelling framework, a more complex pattern of stress propagation. (The principal axes and the propagation characteristics no longer coincide.) The extra fitting parameter provided by OSL is probably not justified by the existing data. One feature of OSL models which stands out strongly (at least in two dimensions) is the presence of reflection and refraction of stress-paths at the central axis of the pile (see Sec. 2.9). Careful experiments on the effect of small perturbing loads could reveal whether or not this really occurs, providing a strong test of this class of model.

In view of its attractive physical features, and of its experimental success, we currently favour the FPA hypothesis as the simplest starting point for more refined theories of sandpiles. It also forms a promising basis for future study of stress propagation in static granular media of geometries quite different from the normal conical pile. The richness of this area is amply illustrated by the handful of examples studied above in

Section 4. Consideration of these and other geometries could allow stringent experimental tests of both the FPA model, and the overall modelling strategy we have proposed. Within this framework, there is, no doubt, scope for much more sophisticated models of how the construction history of a pile determines the local constitutive behaviour, but further efforts in this direction may require much more experimental input. The 
validity of the framework itself deserves close experimental scrutiny, particularly concerning the degree to which RSF scaling is obeyed. Our assumption of scale-free (RSF) behaviour offers an immense simplification, but closer experimental investigation may reveal that this is not quantitative except under some limiting conditions. Despite these uncertainties, we feel that the modelling framework presented above has significant potential to provide improved physical theories of stress propagation granular media.

In future work [22] we will explore the close connection between our OSL model and a recent discrete stochastic models for stress propagation in sandpiles [21] (see also [20]), of which OSL can be viewed as the (mean-field) continuum limit. An important concept arising from the stochastic models and from experiment [20] (see also [47]) is that of stress paths; these are pathways through the medium along which most of the load is locally transmitted. The noise-free models considered in this paper can be viewed as making hypothetical statements about the average orientation and loadbearing properties of these paths (see the discussion of characteristics in Sec. 2.9). Such statements are testable, if not directly, then at least in simulation studies. Ongoing work [26] suggests a promising correspondence between the average alignment of these paths and the orientation angle $\Psi$ arising in the OSL model.

Acknowledgement: The authors are indebted to J.-P. Bouchaud for a series of essential discussions which laid the foundations for this work. We also thank S. F. Edwards, V. Entov, M. Evans, J. Goddard, T.C.B. McLeish, R. M. Nedderman and J. M. Rotter for illuminating discussions. JPW thanks C. E. Lester, C. S. and M. J. Cowperthwaite for supporting enthusiastically this research. MEC acknowledges the hospitality of the Isaac Newton Institute for Mathematical Sciences (Cambridge) where part of this work was done. This work was funded in part by EPSRC under Grant GR/K56223 and in part by the Colloid Technology programme. 


\section{References}

[1] T. Jotaki and R. Moriyama, J. Soc. Powder Technol. Jpn., 60, 184 (1979).

[2] J. Smid and J. Novosad, in Proc. of 1981 Powtech Conference, Ind. Chem. Eng. Symp. 63, D3/V/1-D3/V/12 (1981).

[3] D. C. Hong, Phys. Rev. E., 47, 760 (1993).

[4] J. Huntley, Phys. Rev. E., 48, 4099 (1993).

[5] S. F. Edwards and R. B. Oakeshott, Physica D, 38, 88 (1989).

[6] S. F. Edwards, C. C. Mounfield, Physica A, 226, 1,12,25 (1996).

[7] J.-P. Bouchaud, M. E. Cates, P. Claudin, J. Physique II, 5, 639 (1995).

[8] D. F. Bagster and R. Kirk, J. Powder Bulk Solids Tech., 1, 19, (1985).

[9] K. Liffman, D. Y. Chan and B. D. Hughes, Powder Technology 72, 225 (1992); Powder Technology 78, 263 (1994).

[10] R. .M. Nedderman, Statics and Kinematics of Granular Materials, Cambridge University Press (1992).

[11] V. V. Sokolovskii, Statics of Granular Materials, Pergamon, Oxford (1965).

[12] A. Drescher, Analytical Methods in Bin-Load Analysis, Elsevier, Amsterdam (1991).

[13] D. M. Wood, Soil Behaviour and Critical State Soil Mechanics, Cambridge University Press (1990).

[14] R. A. Bagnold, The Physics of Blown Sand and Desert Dunes, Methuen, London (1941).

[15] J.-P. Bouchaud, M. E. Cates, J. R. Prakash and S. F. Edwards, J. Physique I, 4, 1383 (1994); Phys. Rev. Lett. 74, 1982 (1995).

[16] In Ref.[0], a family of nonlinear models are produced in which a velocity-like parameter $V$ is considered to be a function of the shear stress. The OSL model can be viewed as the limit in which $V\left(\sigma_{z x}\right)$ approaches a step function (giving a positive or negative constant value according to the sign of $x$ ). A finite slope of this function at the origin $\left(V^{\prime}(0) \neq 0\right)$ would lead to rounding of the cusp at the centre of the dip on some characteristic length scale (in violation of RSF scaling).

[17] D. H. Trollope, The Stability of Wedges of Granular Materials, Ph.D. Thesis, University of Melbourne (1956); Proc. 4th Int. Conf. S. M. and F. E., London 2 383 (1957). See also, C. F. Jenkin, Proc. Roy. Soc. A 131, 53 (1931).

[18] D. H. Trollope, in Rock Mechanics in Engineering Practice, (Eds. K. G. Stagg and O. C. Zienkiewicz), Ch.9. John Wiley, NY (1968). 
[19] If support deflection were important, one would not expect RSF scaling as defined below. (The elasticity of the support introduces a length scale just as that of particles would do.)

[20] C. H. Liu, S. R. Nagel, D. A. Schecter, S. N. Coppersmith, S. Majumdar, O. Narayan, T. A. Witten, Science, 269, 513 (1995).

[21] P. G. de Gennes, private communication.

[22] P. Claudin, J. P. Wittmer, J.-P. Bouchaud and M. E. Cates, to be published.

[23] P. A. Cundall and O. D. Strack, Geotechnique 29, 47 (1975); P. A. Cundall, in Micromechanics of Granular Materials, M. Satake and J. T. Jenkins, Eds., Elsevier (Amsterdam) 1987; C. Thornton and D. J. Barnes, Acta Mech., 64, 46 (1986).

[24] V. Buchholtz and T. Poeschel, Physica A, 202, 390 (1994); T. Poeschel and V. Buchholtz, Phys. Rev. Lett., 71, 3963 (1993).

[25] X. Zhuang, A. D. Didwania and J. D. Goddard, J. Com. Phys., 121, 331 (1995).

[26] V. Buchholtz, J.P. Wittmer and M. E. Cates, in preparation.

[27] In principle one could construct piles in which the scaling was nonetheless obeyed, by demanding that the relative variation of the material properties between (say) an element at the apex, and an element directly beneath the apex at the base, must be the same for all piles. However, this would require the spatial gradient of the size distribution, for the element at the base, to vary inversely with the height of the pile. Clearly, the size distribution (and its gradient) in this element is fixed forever long before the eventual height of the pile is decided. We conclude that, although size segregation may arise, models invoking this to explain the dip are inconsistent with the experiments of Ref. [2].

[28] See, e.g., J. Eibl, H. Landahl, U. Haussler and W. Gladen, Beton-und Stahlbetonbau 77, 104 (1982); J. Eibl and F. Dahlhaus, Proc. 3rd Euro. Symp.: Storage and Flow of Particulate Solids, PARTEC Nurnberg, 219 (1995); J. Y. Ooi and J. M. Rotter, Computers and Structures, 37, 361 (1990). J. Y. Ooi, J. F. Chen, R. A Lohnes and J. M. Rotter, Construction and Building Materials, 10, 109 (1996); J. M. Aribert and E. Ragneau; Second European Symposium on Stress and Strain in Particulate Solids, CHISA 90, Paper No 1669, Prague (1990).

[29] See, e.g., J. M. Rotter, J. Y. Ooi, J. F. Chen, P. J. Tiley, I. Mackintosh and F. R. Bennet, Flow Pattern Measurement in Full Scale Silos, British Materials Handling Board Publication, 230 pp (1995) (ISBN 0946637 091); J. Y. Ooi and J. M. Rotter, Proc. Int. Conf. Bulk Materials - Towards the Year 2000, p. 181 (Inst. Mech. Eng., London, 1991); J. Y. Ooi, W. C. Soh, Z. Zhong and J. M. Rotter, Proc. Int. Symp.: Reliable Flow of Particulate Solids II, EFchE Pubs. Ser. No. 96, p.75 (1993).

[30] See e.g.: Y. C. Chen, I. Ishibashi and J. T. Jenkins, Geotechnique, 38, 25, 33 (1988) and references therein; IUTAM Symp. on Deformation and Failure of Granular Materials, P. A. Vermeer and H. J. Luger (Eds.), Balkema, Rotterdam (1982); L. 
Rothenberg, R. J. Bathurst and M. B. Duesseault, in Powders and Grains, Biarez and Gourves, (Eds.), Balkema, Rotterdam (1989).

[31] J. P. Wittmer, P. Claudin, M. E. Cates and J.-P. Bouchaud, An explanation of the central stress minimum in sandpiles, Nature, submitted.

[32] A simple method for determining the stress components in two dimensions on a particular plane through a point and comparing them with the yield criterion is known as Mohr's circle [10]. Unfortunately, this method forces an unusual sign convention we do not wish to introduce here.

[33] C. A. Coulomb, Mem. de Math. de l'Acad. Royale des Sciences 7, 343 (1776).

[34] The tilt axis $\Psi$ exactly bisects the angle between the free surface and the vertical (as proved in Sec. 2.2).

[35] It turns out that discontinuous derivatives can arise for three values of $S$ : at the outer slope $(S=1)$; on the central axis of the pile $(S=0)$; and the point $S=S_{0}$ where the stress information from the apex of the pile reaches the bottom; examples can be seen in Fig.2(a).

[36] Obviously one can equally write the scaling ansatz eqn. (11) as $\sigma=g R Q(\theta)$ where $R$ is the radius measured from the top of the pile and $\theta$ is the angle measured clockwise from the (downwards pointing) z-axis (see Fig.1(c)). This yields the trivial identification $Q(\theta)=g(S) / \sqrt{1+(c S)^{2}}$.

[37] Note that the RSF scaling, while allowing (in two dimensions) a distinction between the construction histories of elements in the right and left halves of the pile, does not permit any distinction between the elements within one half.

[38] It might seem that the problem is overdetermined, but two equations (the first of the eqns. (19) and eqn. (8)) are automatically identical in a radial stress field.

[39] H. A. Janssen, Z. Vert. Dt. Ing. 39, 1045 (1895) .

[40] In the FPA model the tilted axes must be chosen so as to coincide with the principal axes themselves.

[41] The IFE model is not a special case of OSL; however it turns out that the term $\cot (\phi) U$ in eqn. (21) can be checked a posteriori to be rather small for all $S$ and $\phi$. Accordingly, the IFE is well approximated by $C(U)=\eta+\lambda U^{2}$ with no term linear in $U$, and $\lambda>0$ (leading to a quadratic hump, as discussed by BCC). This means that IFE is in some sense "close" to the BCC model on the $\eta, \mu$ plane (hence its smooth behaviour at the central axis) even though it lies outside the OSL parameterization.

[42] Though an analytic proof should be possible, we have so far only checked this numerically. 
[43] The "Coulomb yield criterion" is the frictional analogue of Tresca's criterion 13. The frictional analogue of von Mises' criterion is the "Conical yield criterion". For a two dimensional problem both criteria are identical. In three dimensions, the failure conditions predicted by these two criteria only differ slightly and the stresses (calculated for instance with an IFE assumption) are predicted to be similar $[10$.

[44] One argument for this is that grains at the surface cannot be pressed together more in $\chi$ direction than in $r$-direction: $s_{r r}(1) \geq s_{\chi \chi}(1)$.

[45] We are grateful to Prof. V. Entov for suggesting this thought-experiment.

[46] J. M. Rotter, private communication.

[47] S. R. Nagel, Rev. Mod. Phys,. 64, 321 (1992). 
Figure 1: The symmetrical sandpile. (a) Definition of the normal construction history of a pile. The grains fall down from the point source on the pile and roll down the slopes, which are at the repose angle $\phi$. (b) The arching concept. In the EdwardsOakeshott formulation the weight supported at a point the base is proportional to the length of the arch impinging on that point. Outer (incomplete) arches are unstable. (c) Coordinates for the 2-d sandpile. The scaling variable $S=r /(c z)$ is unity on the free surface. The height of this pile is $H$. The $(z, r)$ coordinates, and also a second set $(n, m)$ rotated through angle $\tau$ are shown. The ellipse denotes the stress tensor whose major axis is inclined at angle $\Psi$ to the vertical in the neighbourhood shown. (d) Cylindrical polar coordinates for the 3 -d pile.

Figure 2: Results for a two dimensional symmetrical sandpile with a surface obeying IFS; $\phi=30^{\circ}$. (a) Reduced shear stress $s_{z r}$ and reduced vertical normal stress $s_{z z}$ as a function of scaling variable $S=r /(c z)$. Results for the IFE model (found numerically), the BCC model, and the FPA model are compared. Those for the third stress component $s_{r r}$ are not shown but can be deduced from those given via the appropriate constitutive equation in each case. (b) The same comparison, showing instead the yield function $\Upsilon(S)$. For IFE this is unity everywhere by definition; for FPA and BCC in two dimensions it is unity throughout the "outer" regime of the pile (the same does not apply in 3-d). In the FPA model the stress at the centreline is isotropic and $\Upsilon=0$ there. (c) The same comparison, showing now the orientation angle $\Psi$ of the major principal axis. At the free surface, where $\Upsilon=1, \Psi$ bisects the free surface and the vertical: $\Psi=\psi$ (which is $30^{\circ}$ in this case). The same relation holds everywhere in FPA; in BCC it holds only in the outer regime. In the IFE model, it holds at the surface only. The "shooting" to the surface value in the numerical solution of IFE is not perfect because of the numerical instability generated by the singularity on the surface.

Figure 3: Sketch of the geometry of the FPA model. The stress ellipsoid has fixed inclination angle $\Psi=\psi$; its ellipticity varies from zero at the centre of the pile to a maximum in the outer region. The outward and inward stress propagation characteristics are indicated by short-dashed and long-dashed lines; these are at rightangles and coincident with the principal axes of the stress ellipsoid.

Figure 4: The $(\mu, \eta)$-plane for OSL model parameters. For a normal pile these must lie on the IFS line, shown as a full line for $\phi=30^{\circ}$ and dashed for $\phi=10^{\circ}$. The BCC model (open symbols) and FPA model (filled symbols) are marked on the IFS line in each case. Note that, on this plot, BCC models (for different $\phi$ ) all lie on the vertical $\mu=0$ axis, separating models showing a dip $(\mu<0)$ from those with a hump. Likewise FPA models (for different $\phi$ ) all lie on the horizontal $\eta=1$ axis, separating models active near the centre of a pile (below the axis) from those which are passive there $(\eta>1)$. Dash-dotted lines denote the zone within which the solutions obey the Coulomb yield requirement; the left boundary (marked $R_{P}$ ) denotes passive failure at the centre of the pile and the right $\left(R_{A}\right)$ active failure there. 
Figure 5: Construction of the solution of the OSL model using characteristics. (a) The response to a point source is constructed as three rays consisting of delta functions of the amplitude shown. Reflection and refraction of the rays at the centreline occurs when the two wave speeds, $c_{1}$ and $c_{2}$, are not equal in magnitude. (b) The solution for a symmetric pile is constructed by summing over all sources whose rays end in a short segment $\Delta x$ at the base of the pile. This defines four strips of material, as shown, of lengths $L_{1-4}$ and widths $w_{1-4}$. Multiplying the area of each strip by the appropriate amplitude factor, and adding, gives the piecewise linear solution for the vertical normal stress. Similar solutions for the other stresses are likewise obtained (using the ratios of stress components within each ray as given in the text).

Figure 6: Results in three dimensions for the FPA model for $\phi=33^{\circ}$ with secondary closures $D_{1}$ and $D_{2}$ defined in the text. Also shown are the data of Ref. [2 for quartz sand (closed symbols) and NPK-1 fertilizer (open symbols), both of which have repose angles of $33 \pm 1^{\circ}$. Though departures are apparent near the maximum of the vertical stress, the dip is reproduced satisfactorily; there are no adjustable parameters in the FPA model. The difference between the two secondary closures is similar to that between the two materials, though we attach no special significance to this. Note the data collapse from piles of different heights; this confirms that RSF scaling is obeyed to experimental accuracy.

Figure 7: Comparison of different primary closures with the same secondary closure relation $D_{1}$, for $\phi=33^{\circ}$. The IFE and BCC models, which do not give a dip, are clearly ruled out by the data of Fig.6. However, it is harder to distinguish OSL models with the adjustable $\eta$ parameter in the range $0.8<\eta<1.2$ from the FPA model which has $\eta=1$. A parameter values different from 1 cannot be ruled out, but nor does one seem to be supported by the data of Fig. 6 .

Figure 8: The yield function $\Upsilon$ as a function of scaling variable $S$ for the FPA, IFE and BCC models. Note that for FPA and BCC, $\Upsilon$ now saturates the Coulomb condition, $\Upsilon=1$, only at the free surface and not through a finite part of the pile. The apparent cusps on the FPA and BCC curves are numerical artefacts arising from the shooting procedure used to solve the equations.

Figure 9: A pile whose shape is changed after being constructed normally. (a) Geometry of the altered pile; dashed lines show the major principal axis orientation, which is unaffected by the removal of grains above. (b) Resulting stress distribution compared with a pile whose repose angle is the same as the final one of the altered pile. (Initial slope $\phi=30^{\circ}$, final slope $20^{\circ}$. ) (c) The application of a tilt $t$; (d) the final "repose" angle $\alpha+t_{\max }$ of the tilted pile, plotted against $\phi$. This is determined by finding the maximum $\alpha$, given $t$, for which the pile remains within the Coulomb yield threshold. For reasonable $\phi$, and small $t$, the final repose angle is almost the same as that of a normal pile. 
Figure 10: Construction of an asymmetric pile from one half of a normal pile. (a) Geometry of the altered pile; dashed lines show the major principal axis orientation, which is unaffected by the removal of grains above. The newly created pile has axes uniform throughout, rather than discontinuous at the centre line. This alters the stress propagation behaviour. (b) The maximum $\beta$ that can be chosen in the geometry of (a), as a function of $\phi$, to avoid violation of Coulomb's yield condition in the newly formed pile. According to the FPA model, $\beta_{\max }$ is quite different from the ordinary repose angle $\phi$. (c) Resulting stress distribution and yield function under the new pile (the scaling variables are the same as for the unmodified pile). The apex of the new pile is marked with an arrow. There is now a lopsided hump, rather than a dip, in the vertical normal stress.

Figure 11: Construction of an asymmetric pile from both halves of a normal pile, leaving the apex in the same position. (a) Geometry of the altered pile; dashed lines show the major principal axis orientation, which is unaffected by the removal of grains above. (b) Resulting stress distribution and yield function under the new pile (the scaling variables are the same as for the unmodified pile). The apex of the new pile is marked by singularities in the vertical normal stress and in the yield function, for the reasons discussed in the text. For the parameters shown, the dip is present in one half of the pile but not the other.

Figure 12: Vertical normal stress beneath a symmetric pile made normally, of height $H$, from which an upper pile of height $z_{c}$ has been removed. The dip is progressively eliminated as material is taken away from the upper part of the pile. 


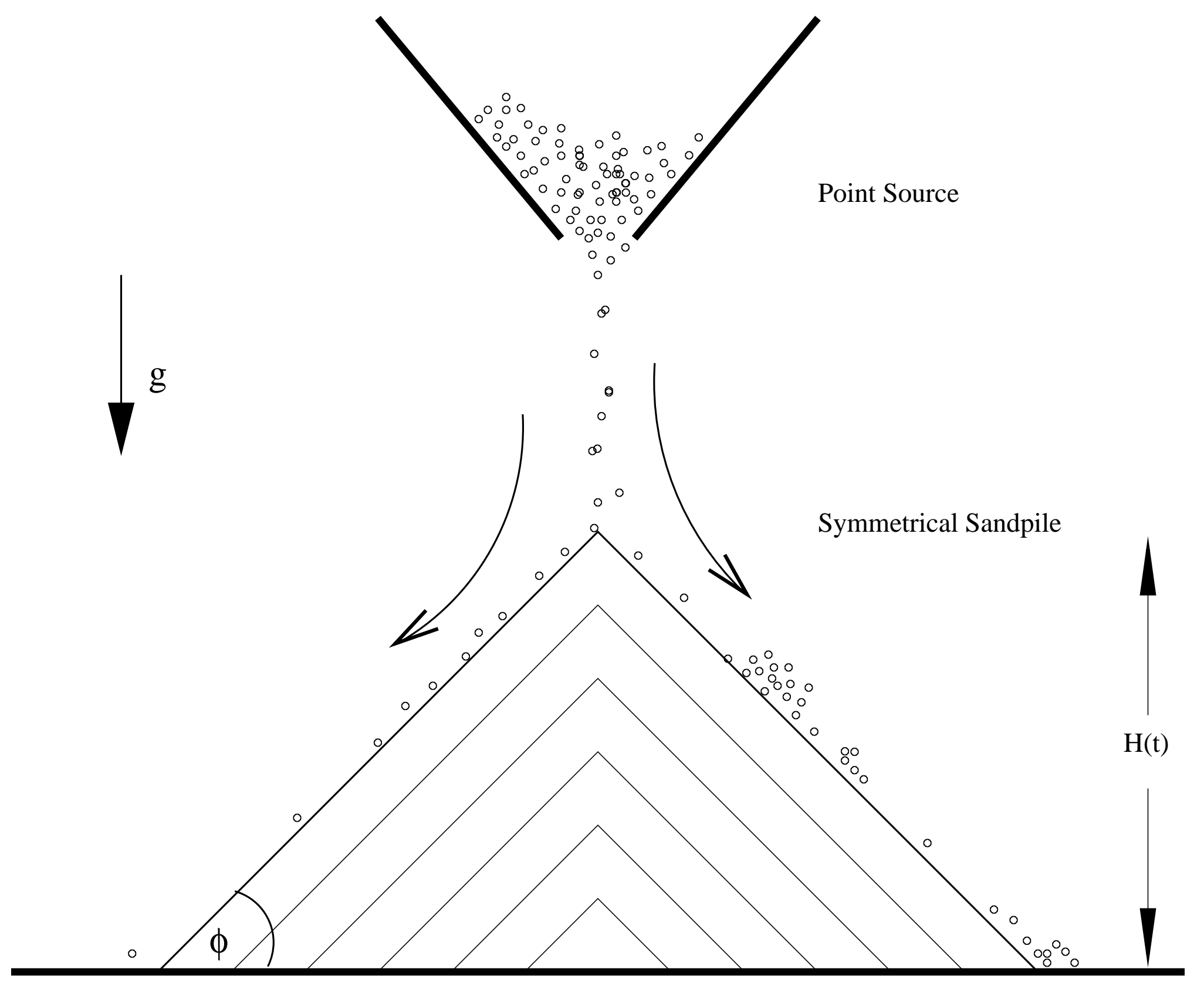



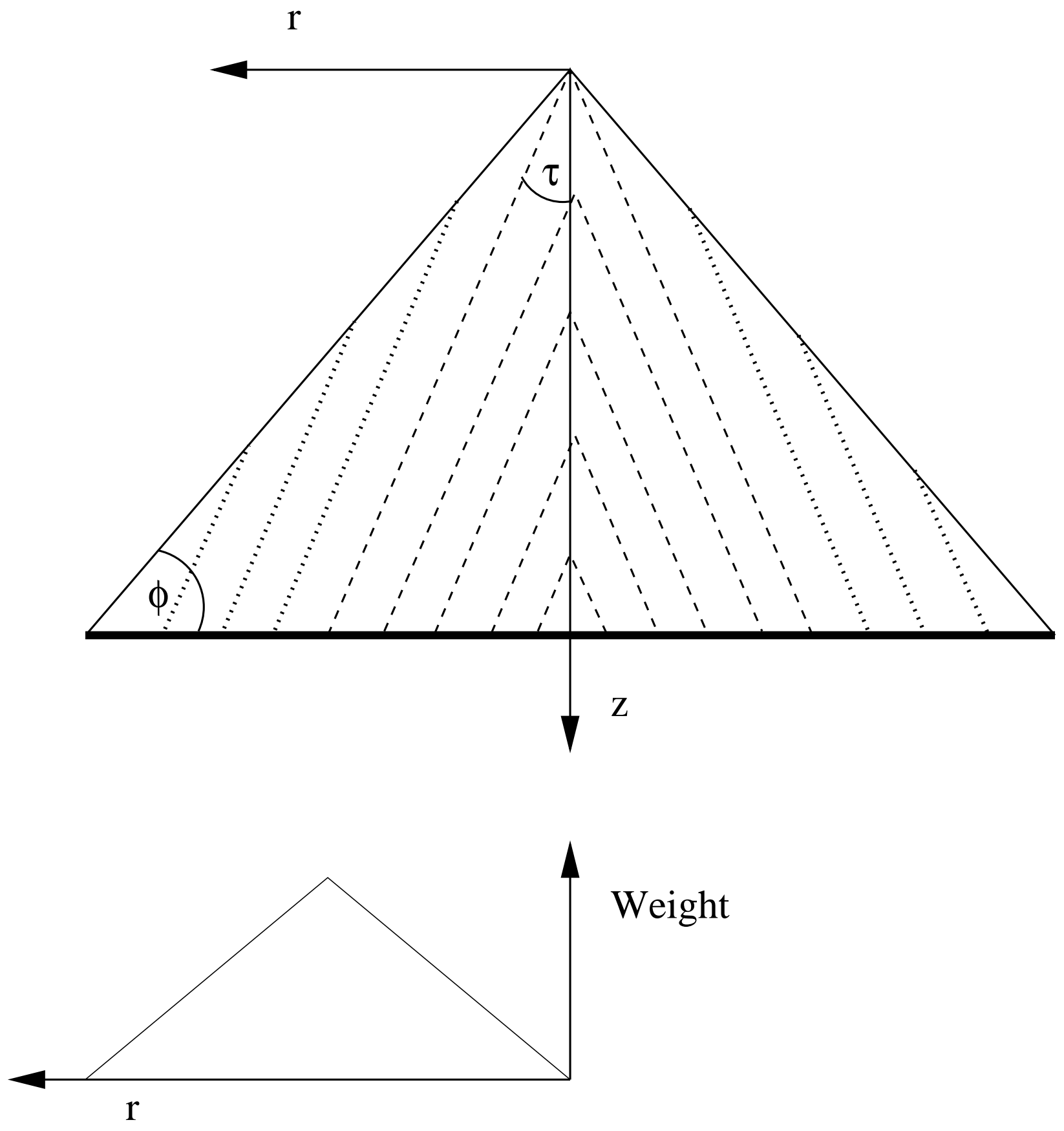


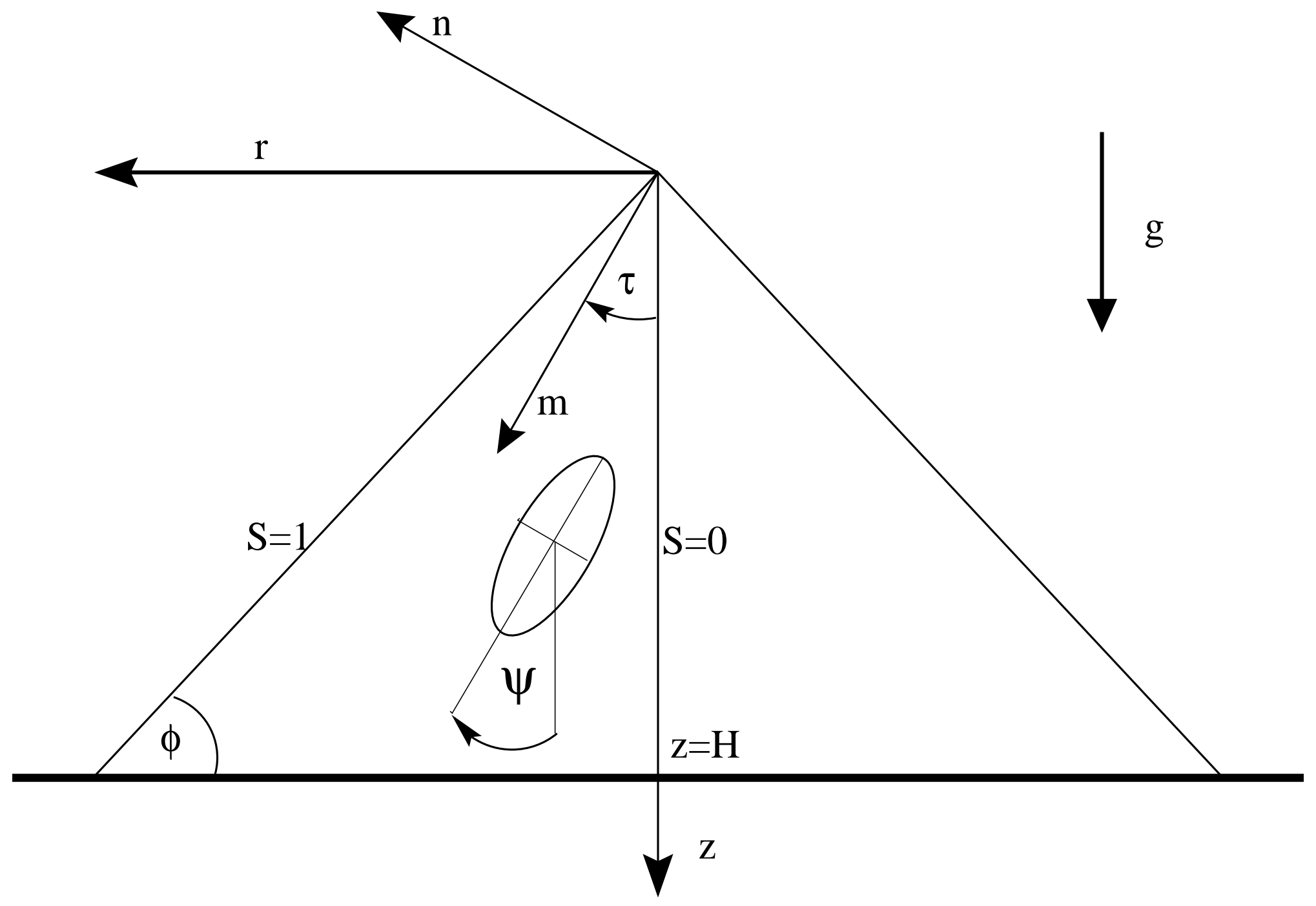




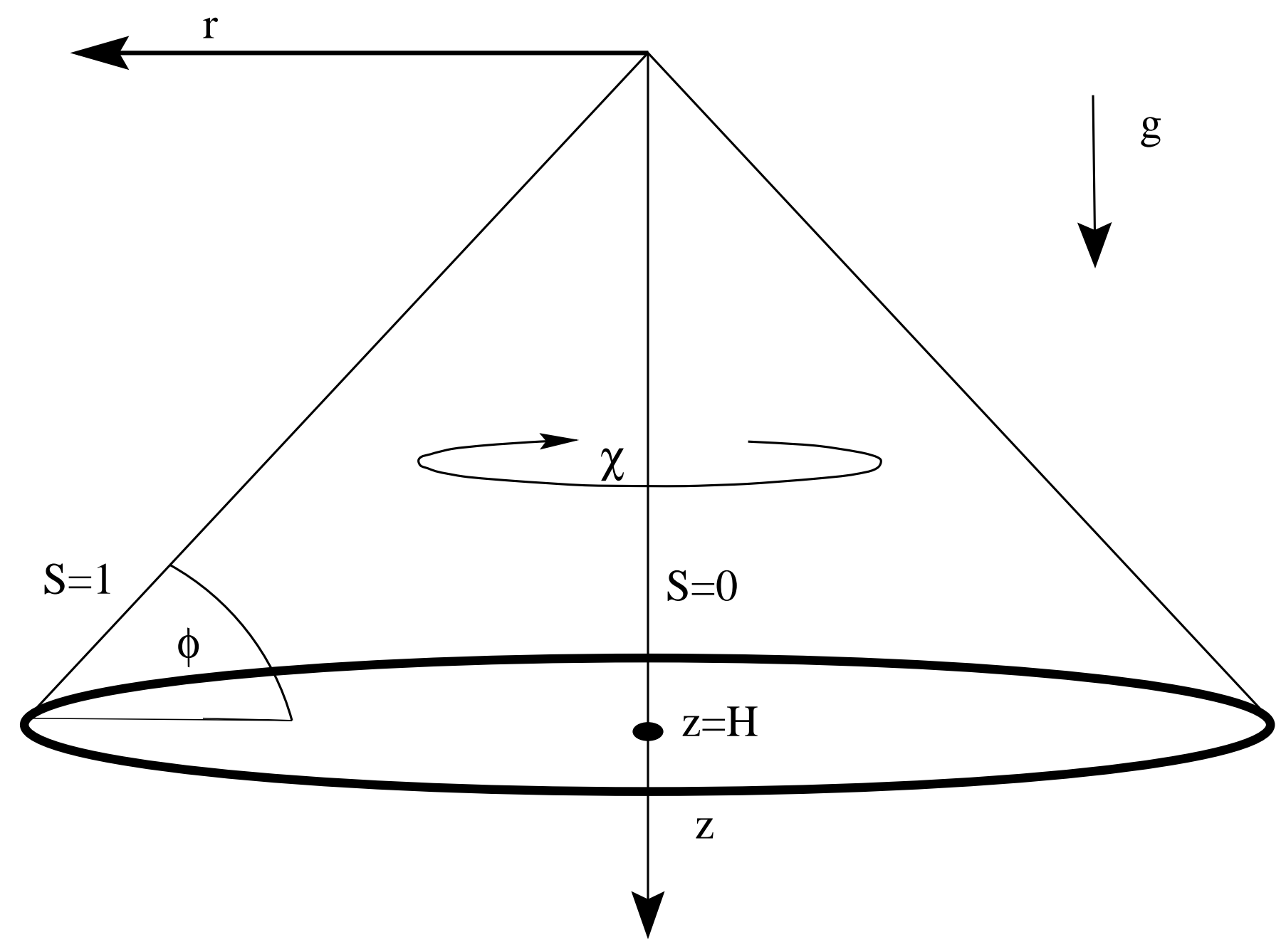




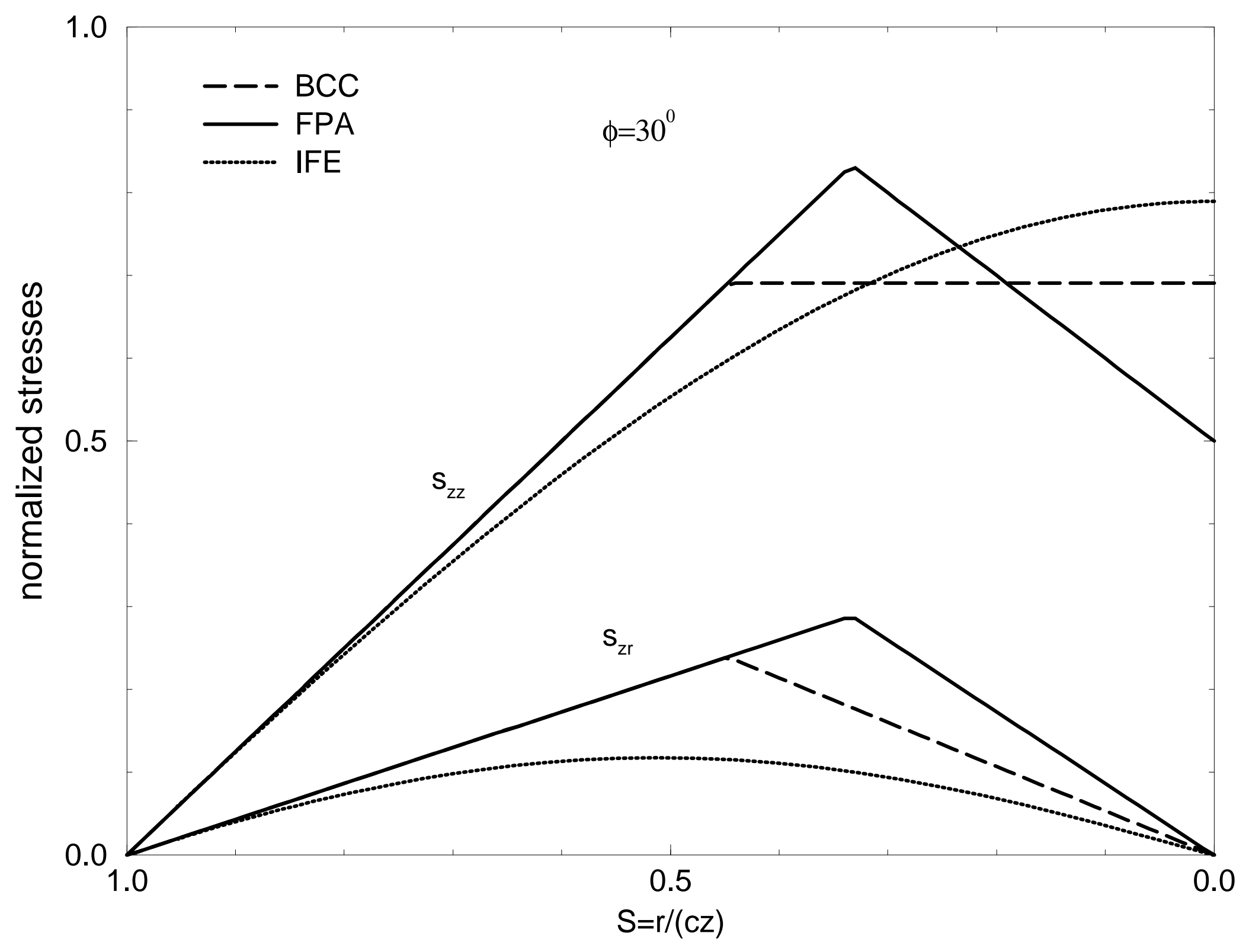




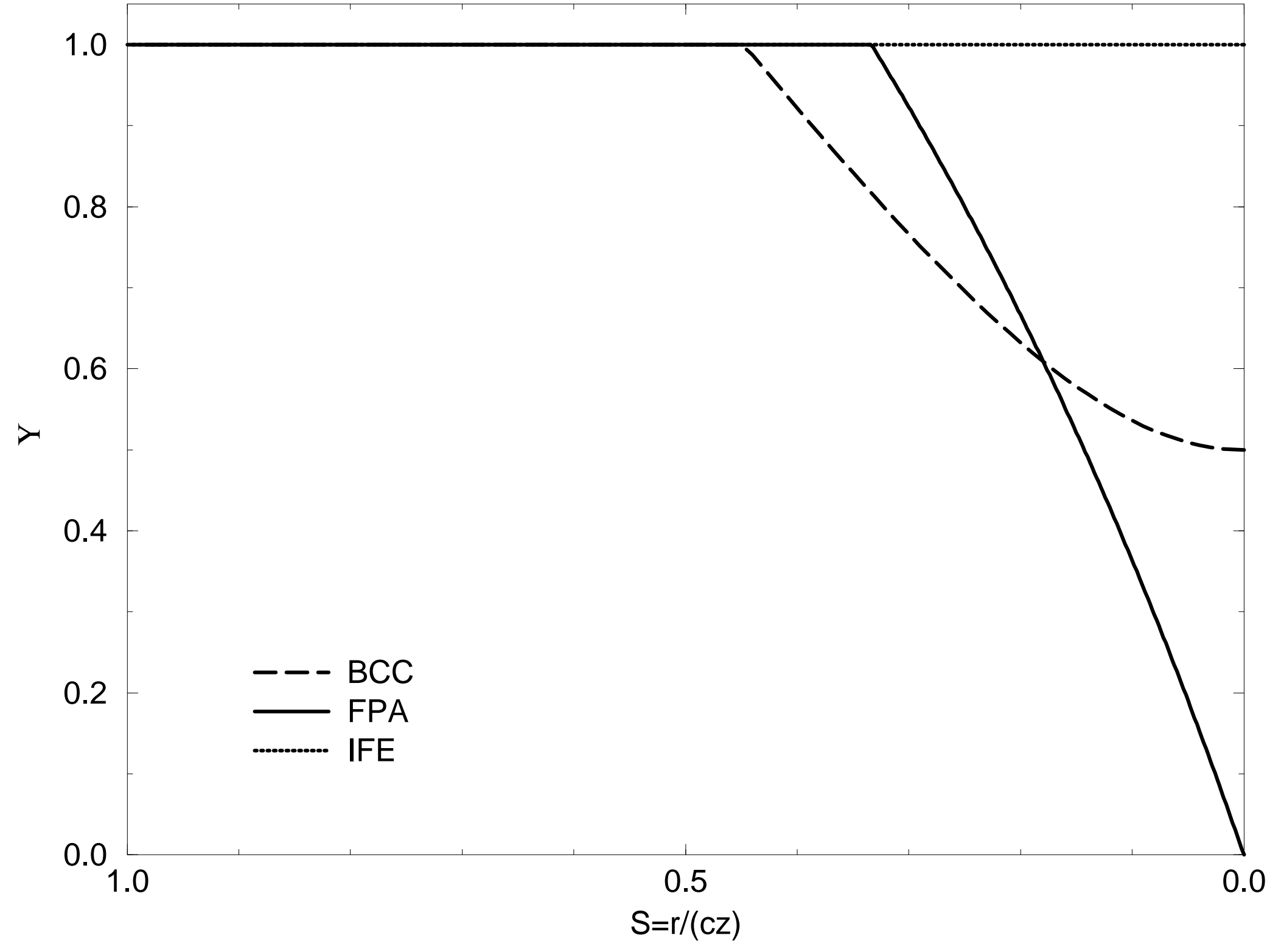




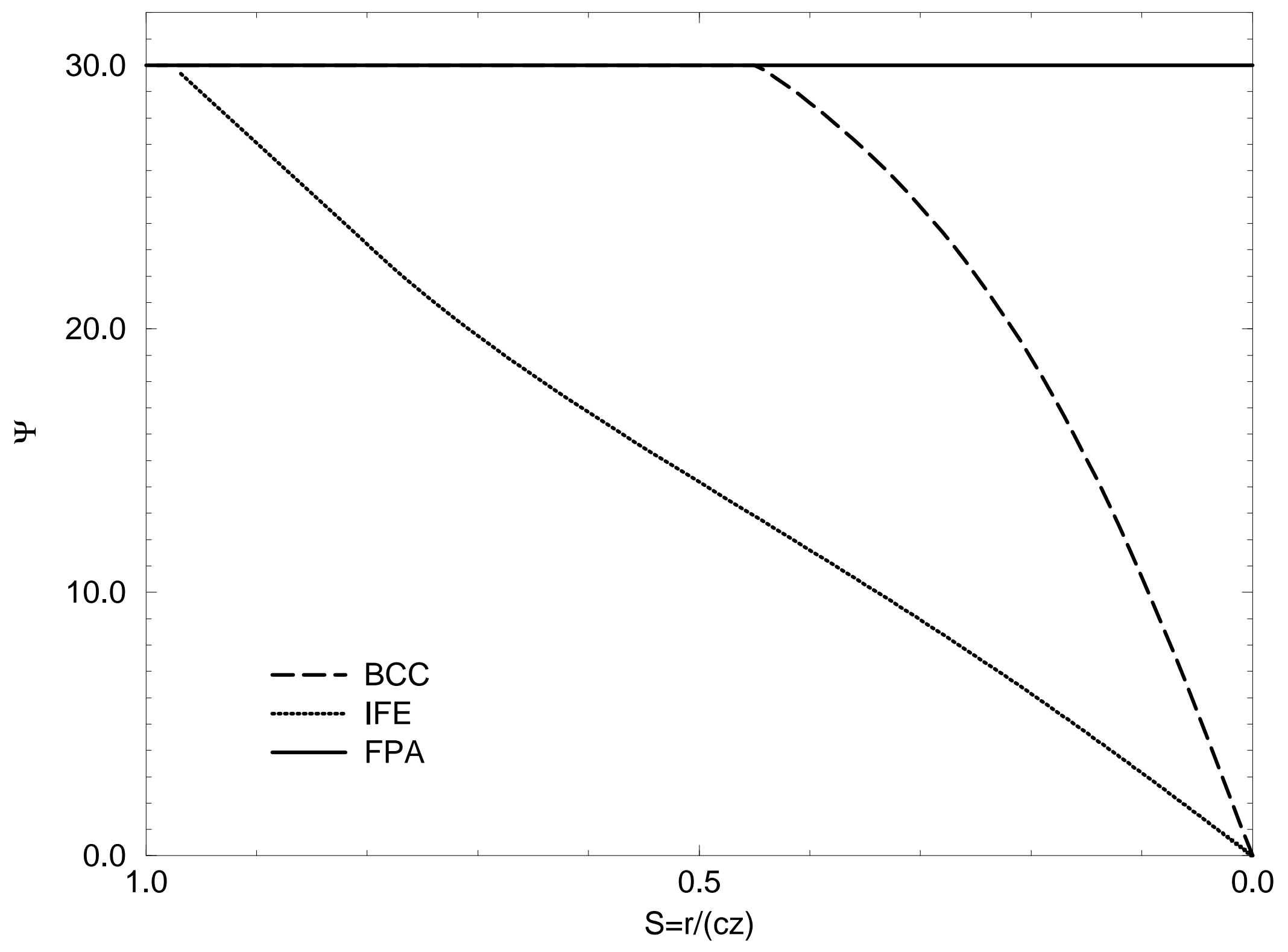




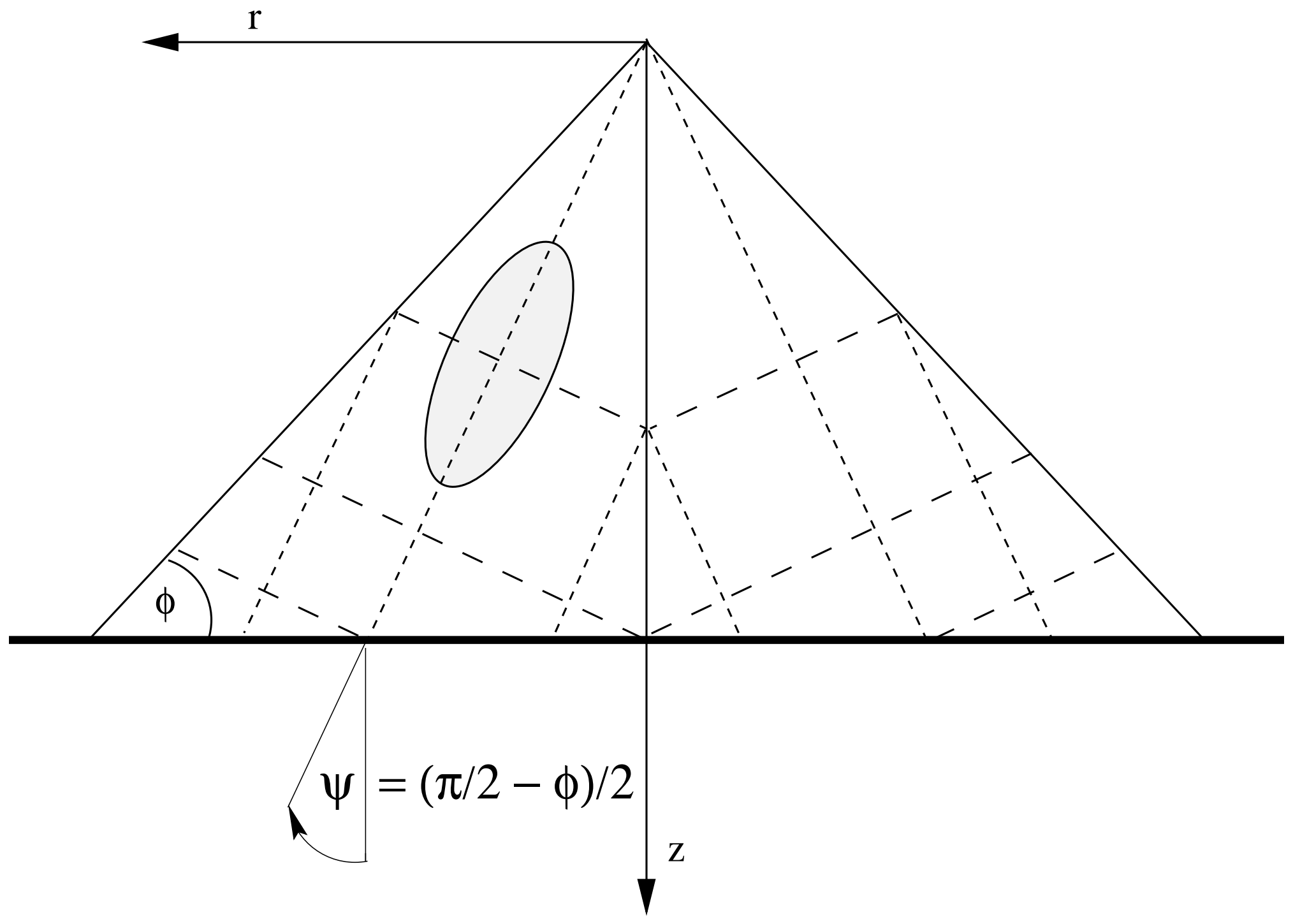




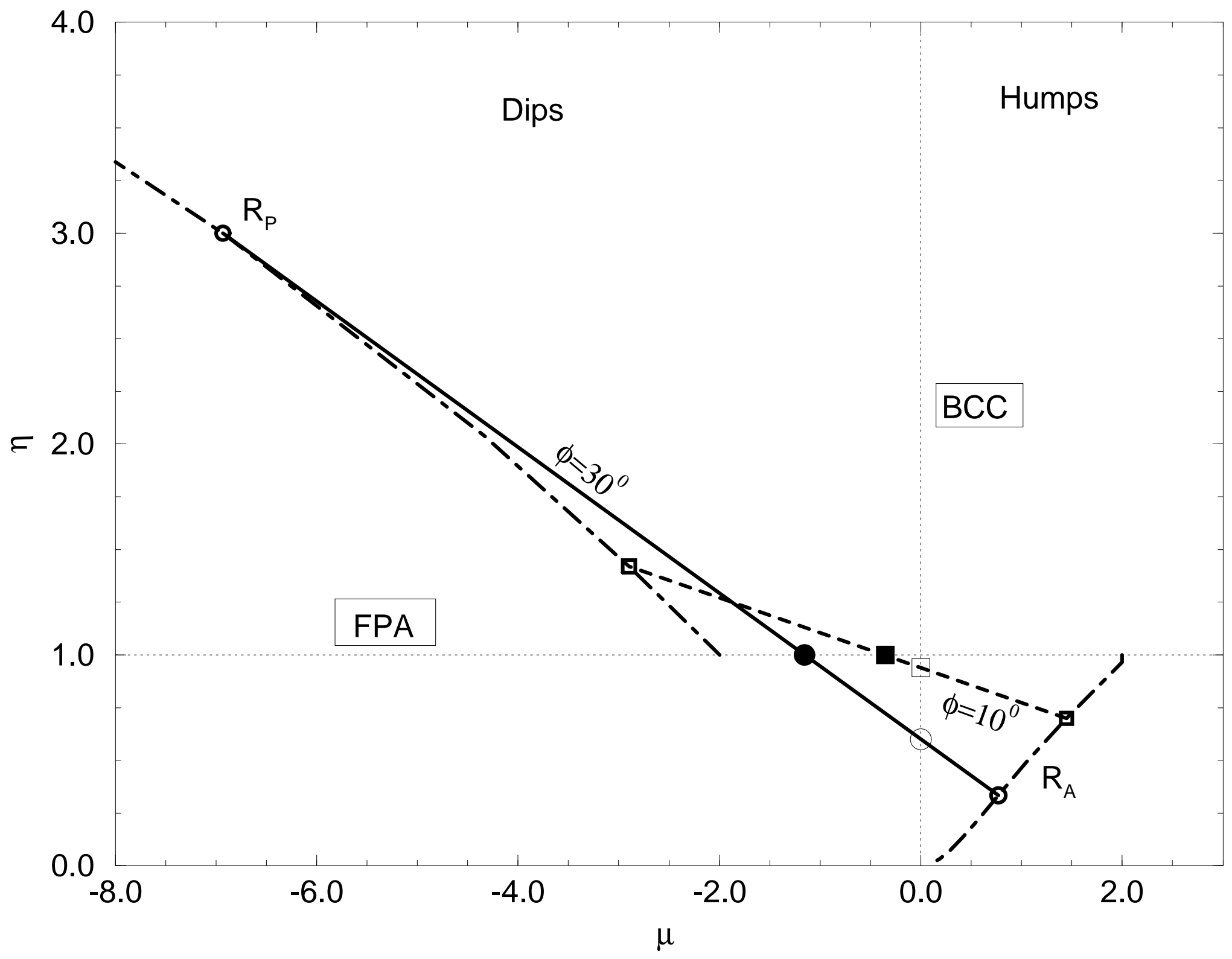




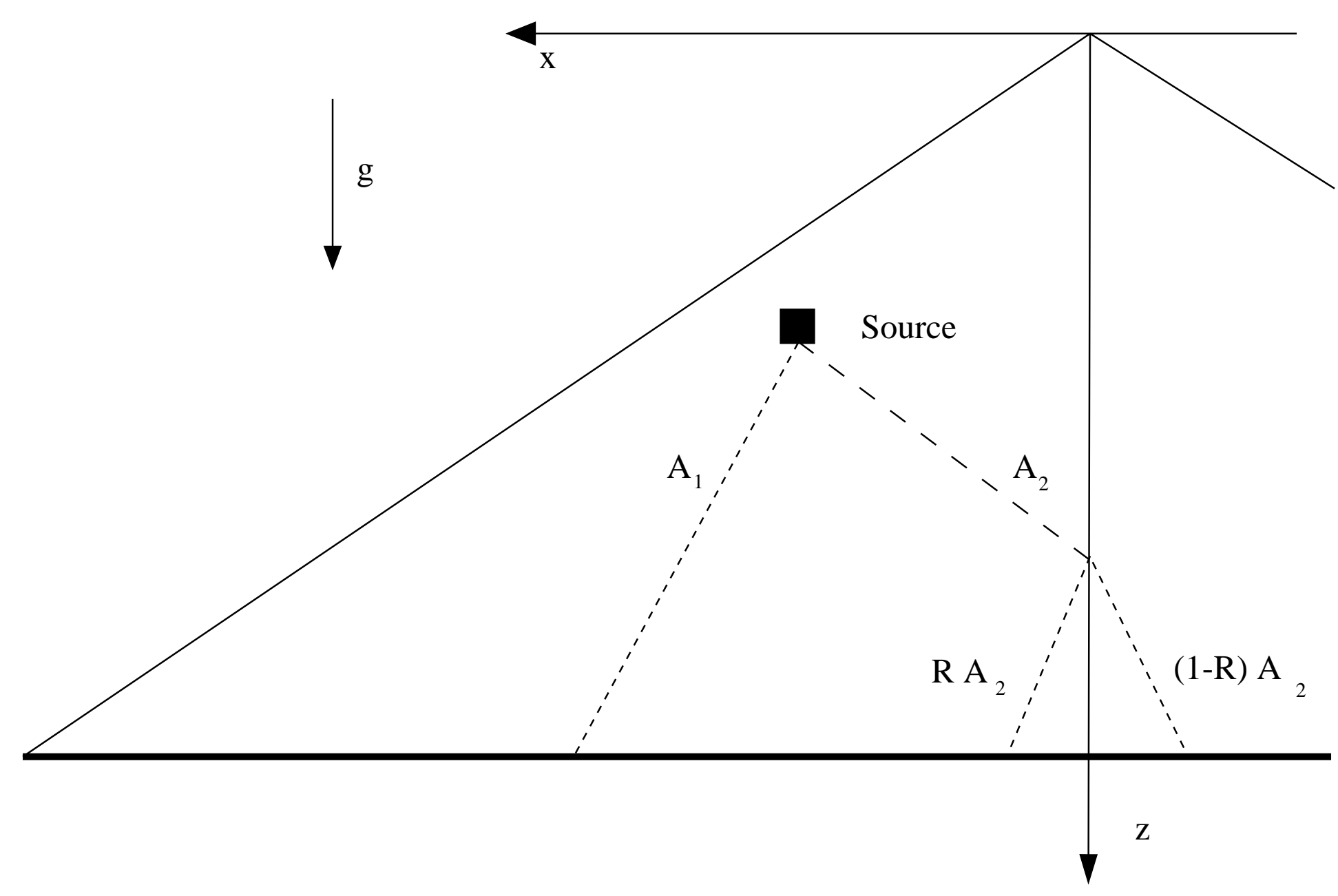




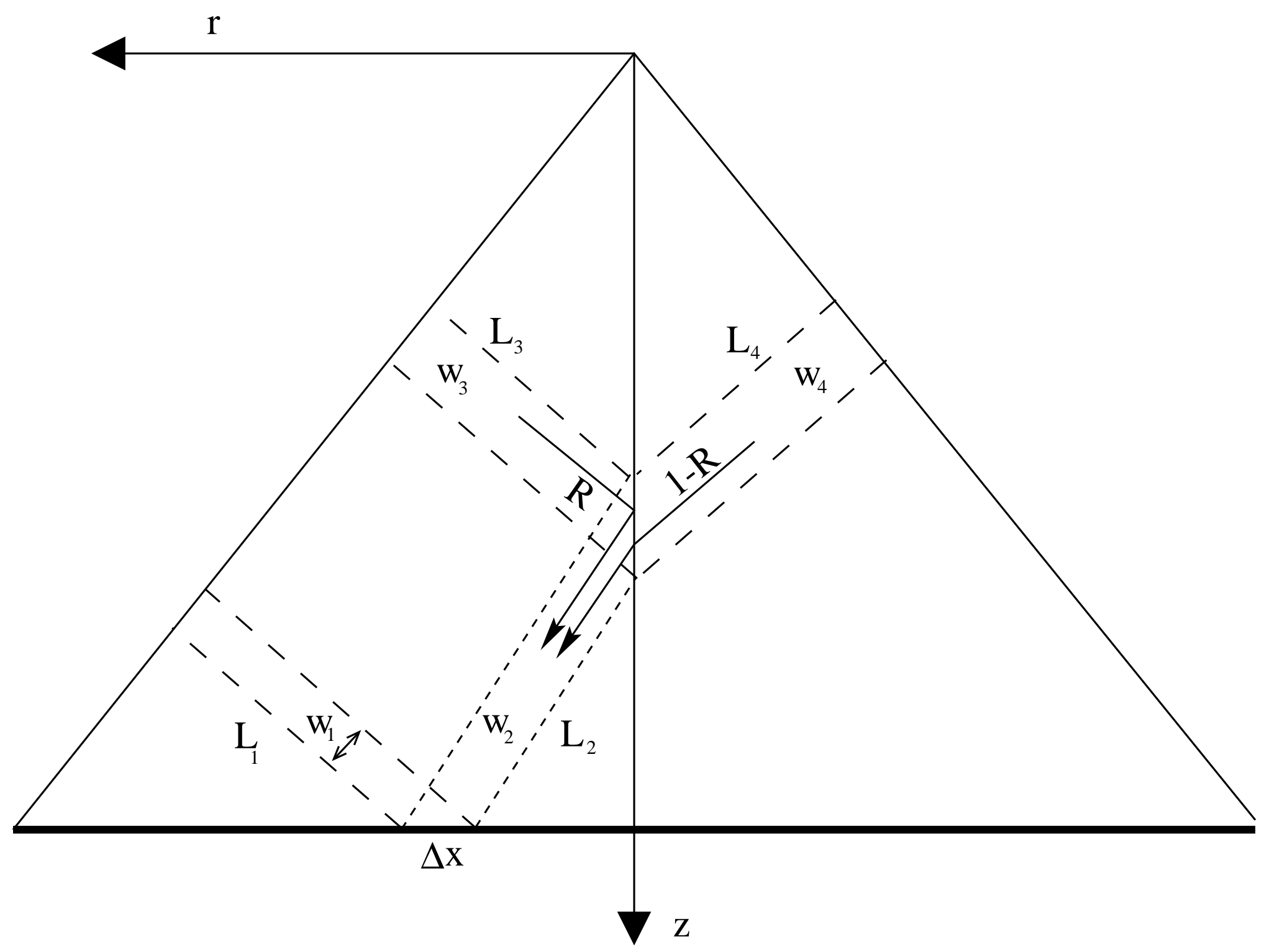




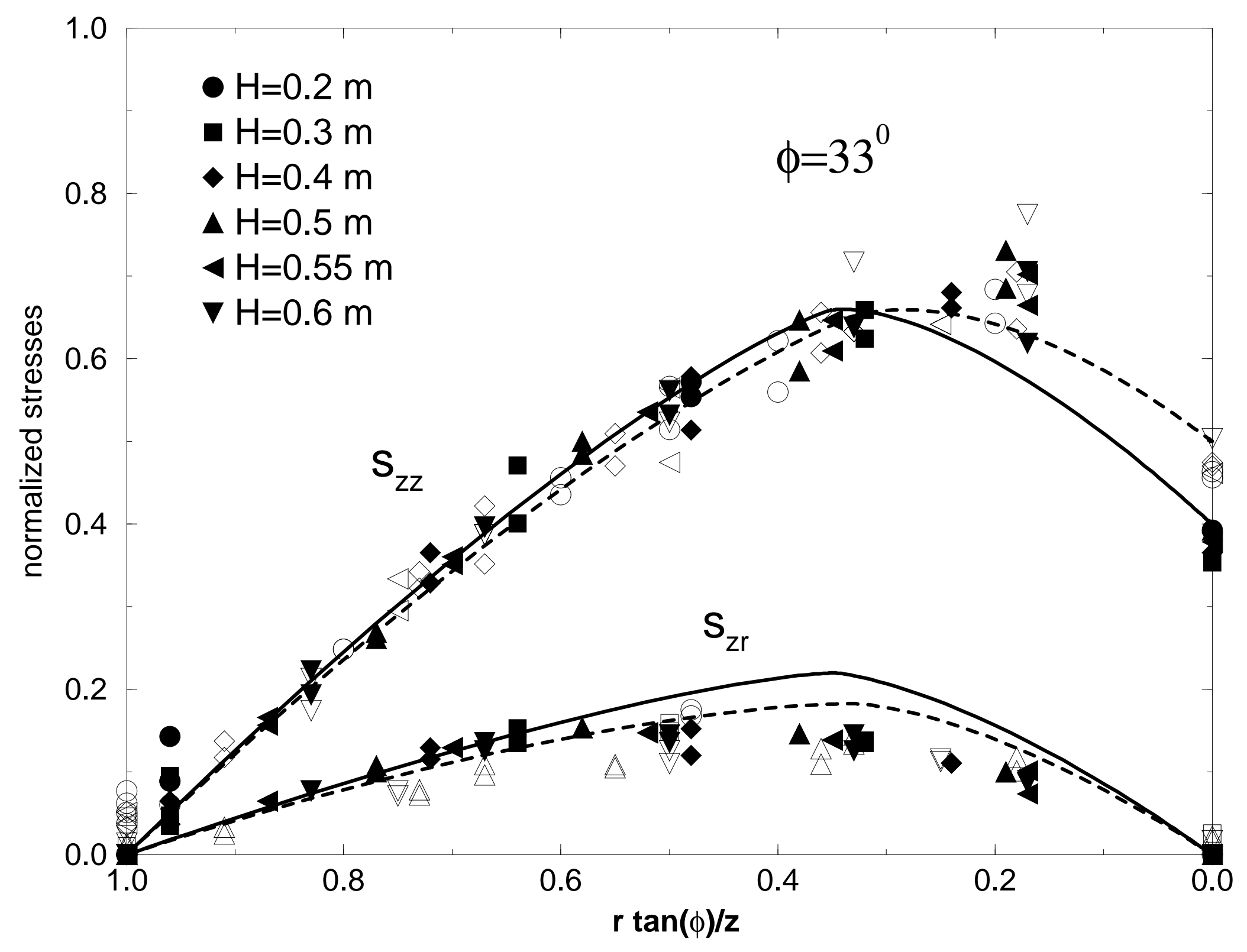




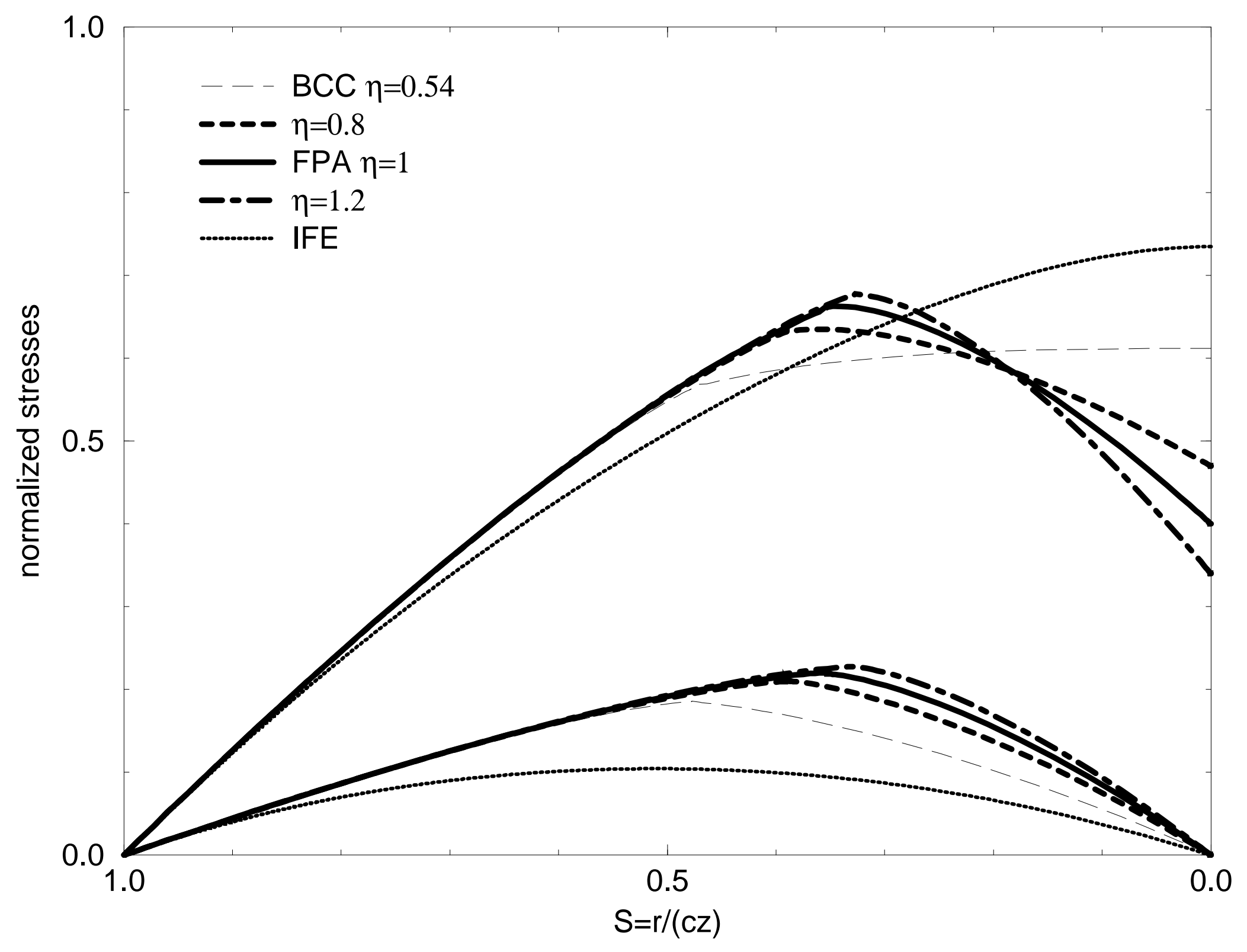




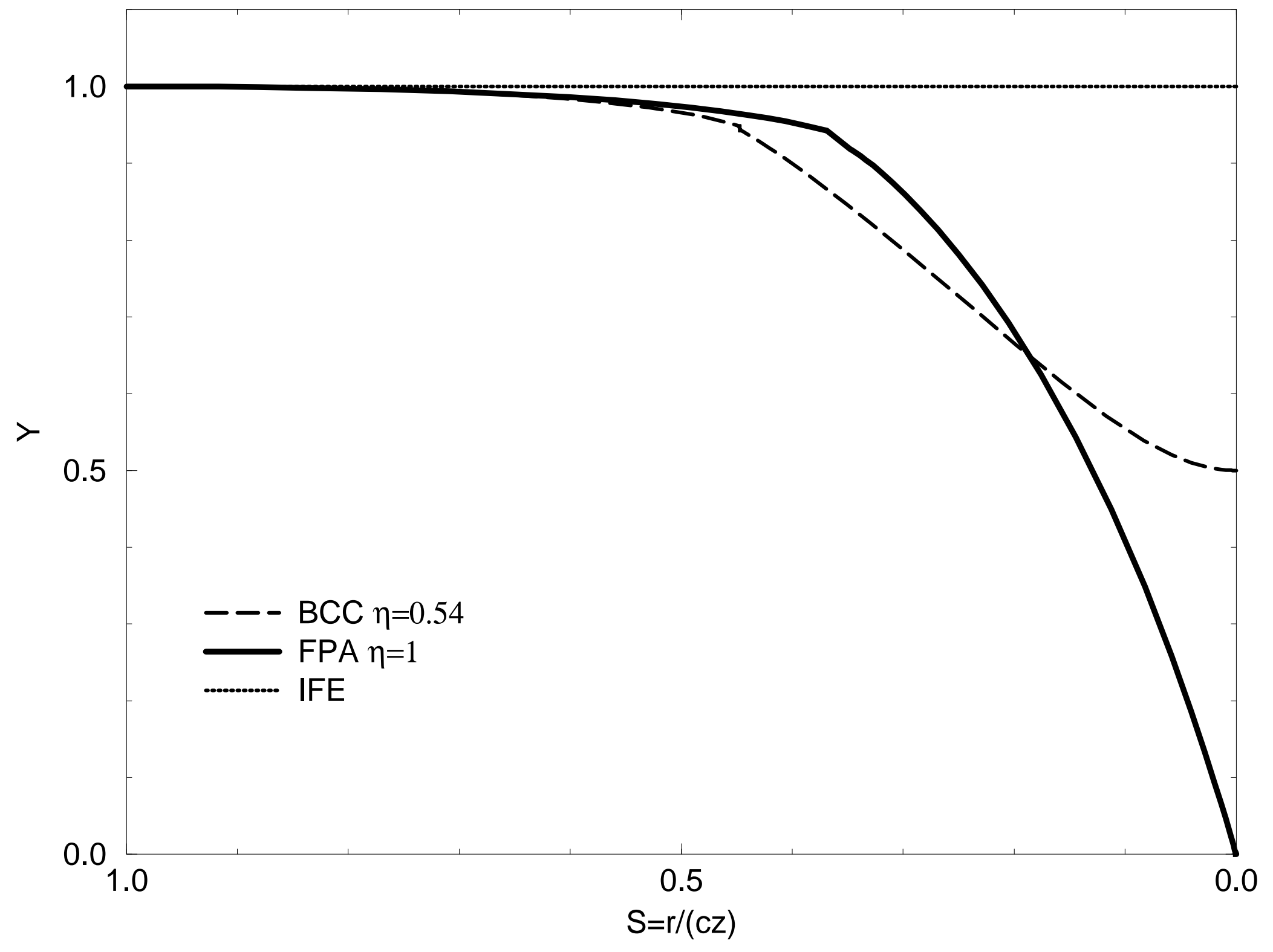




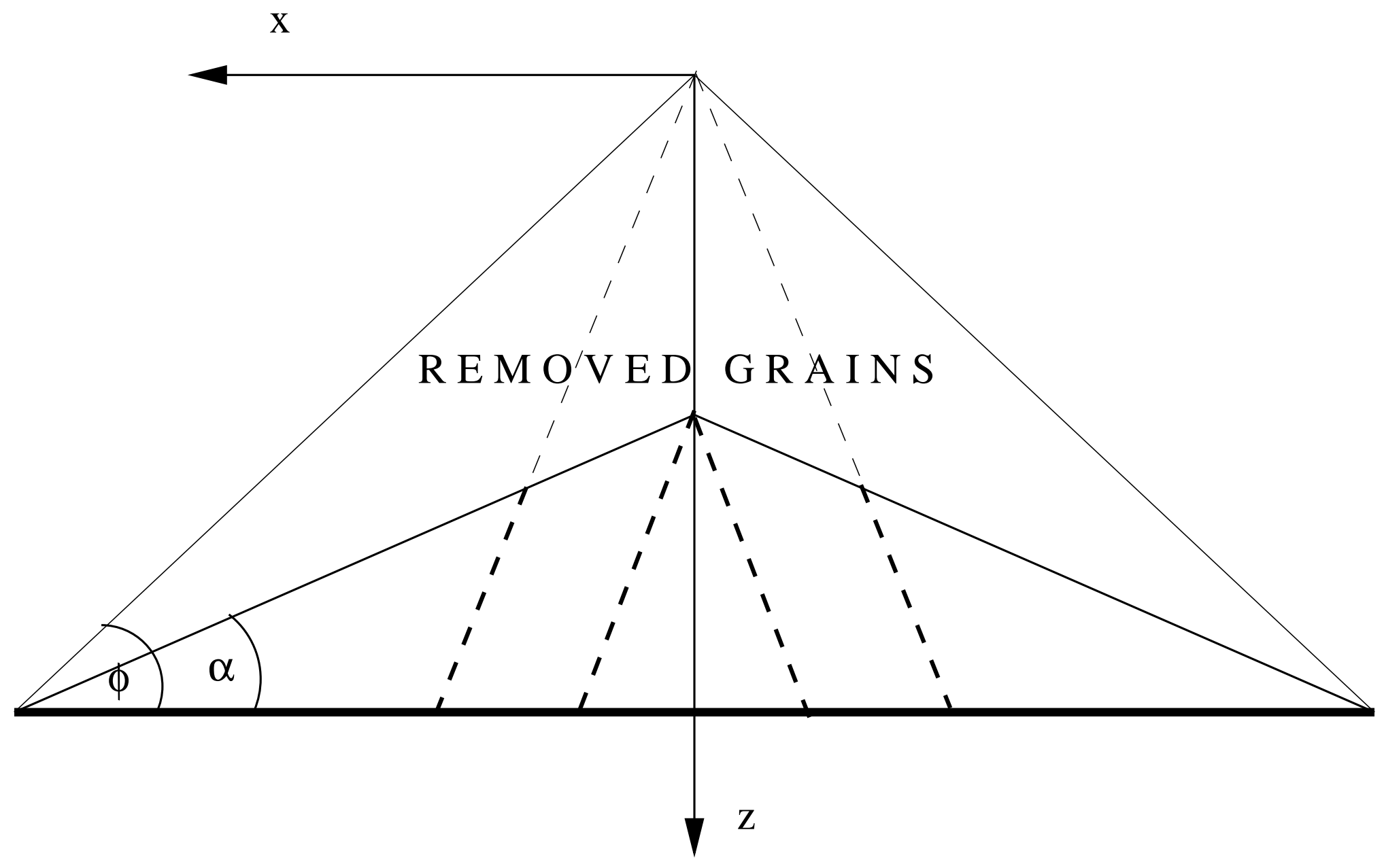




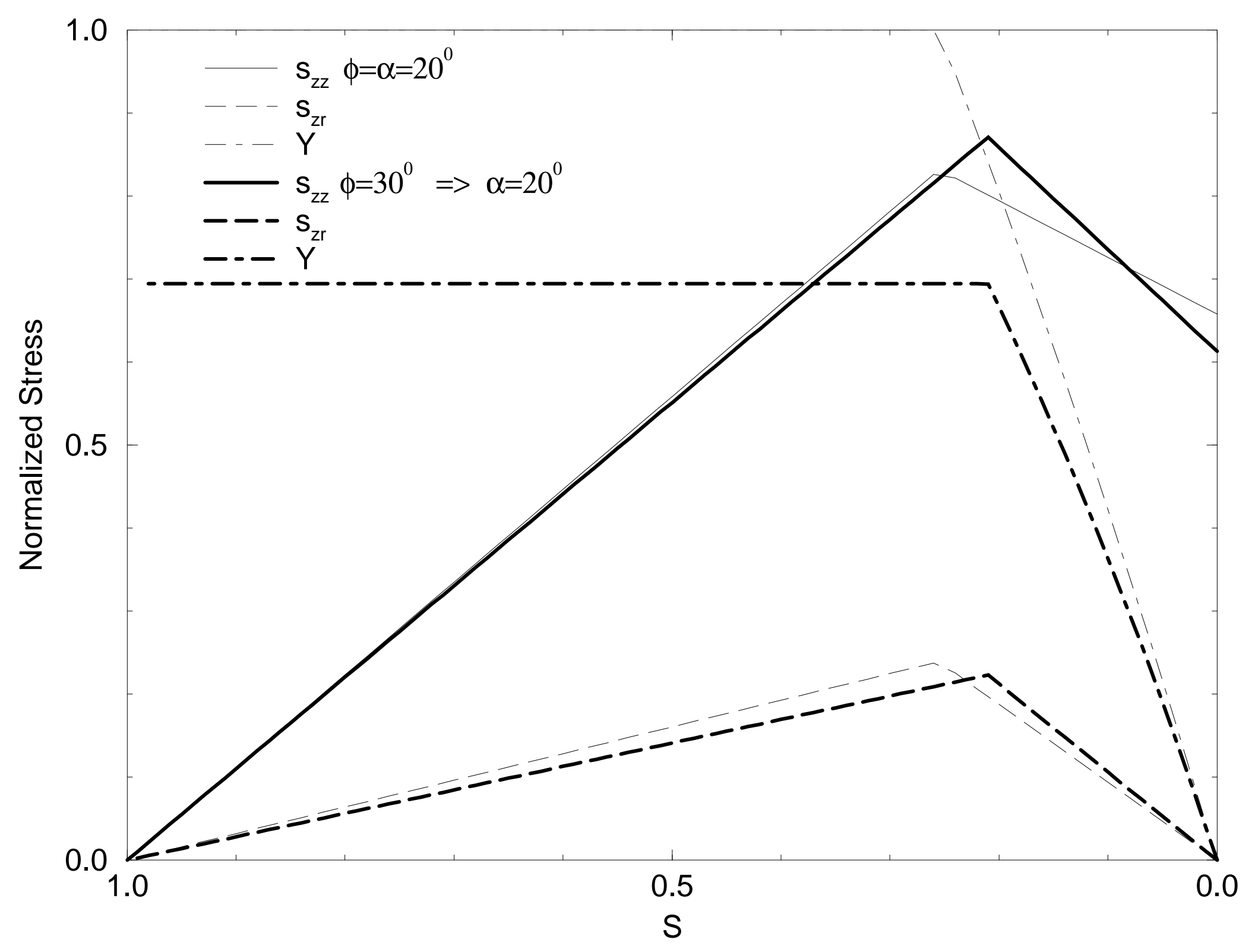




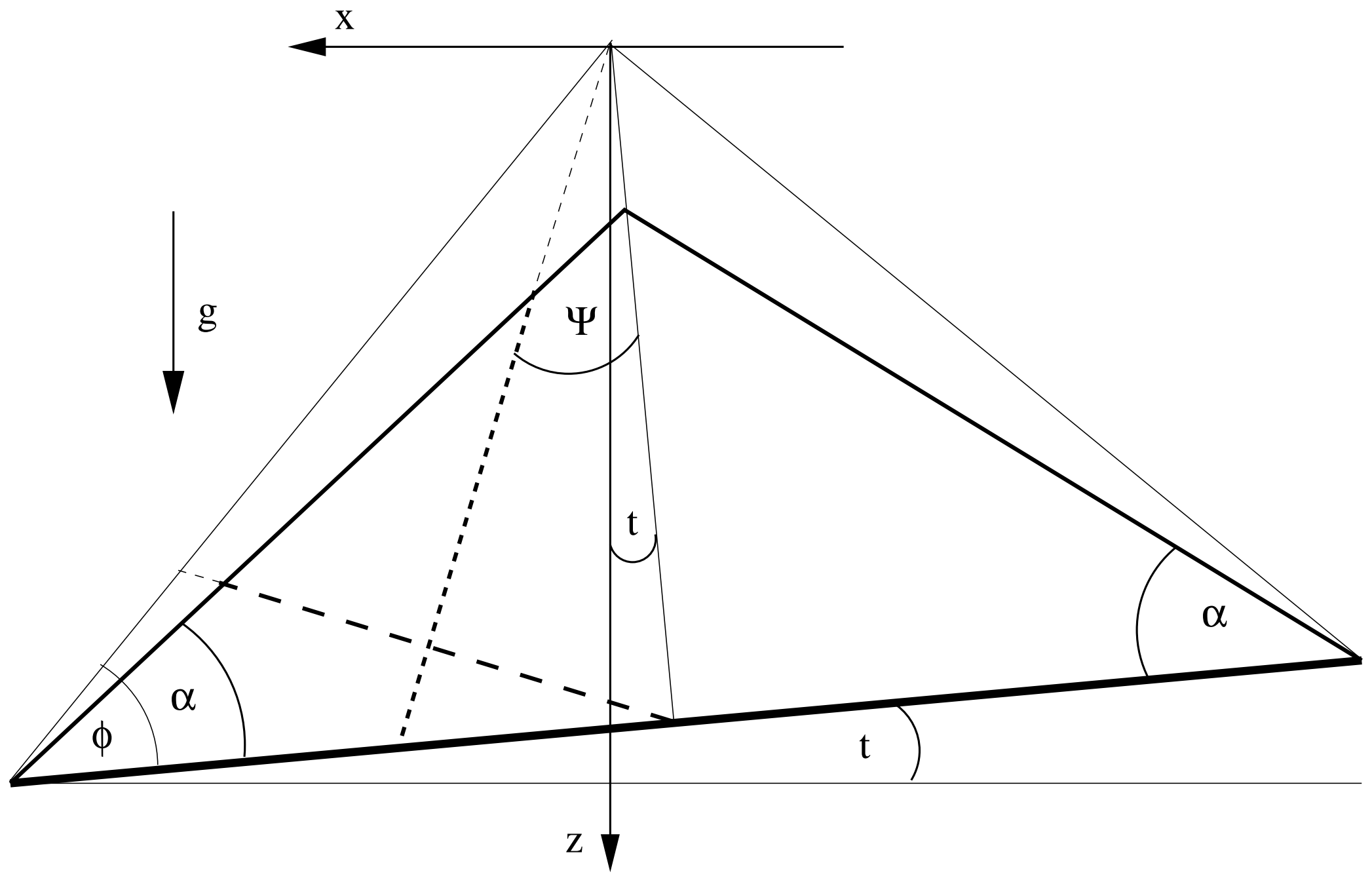




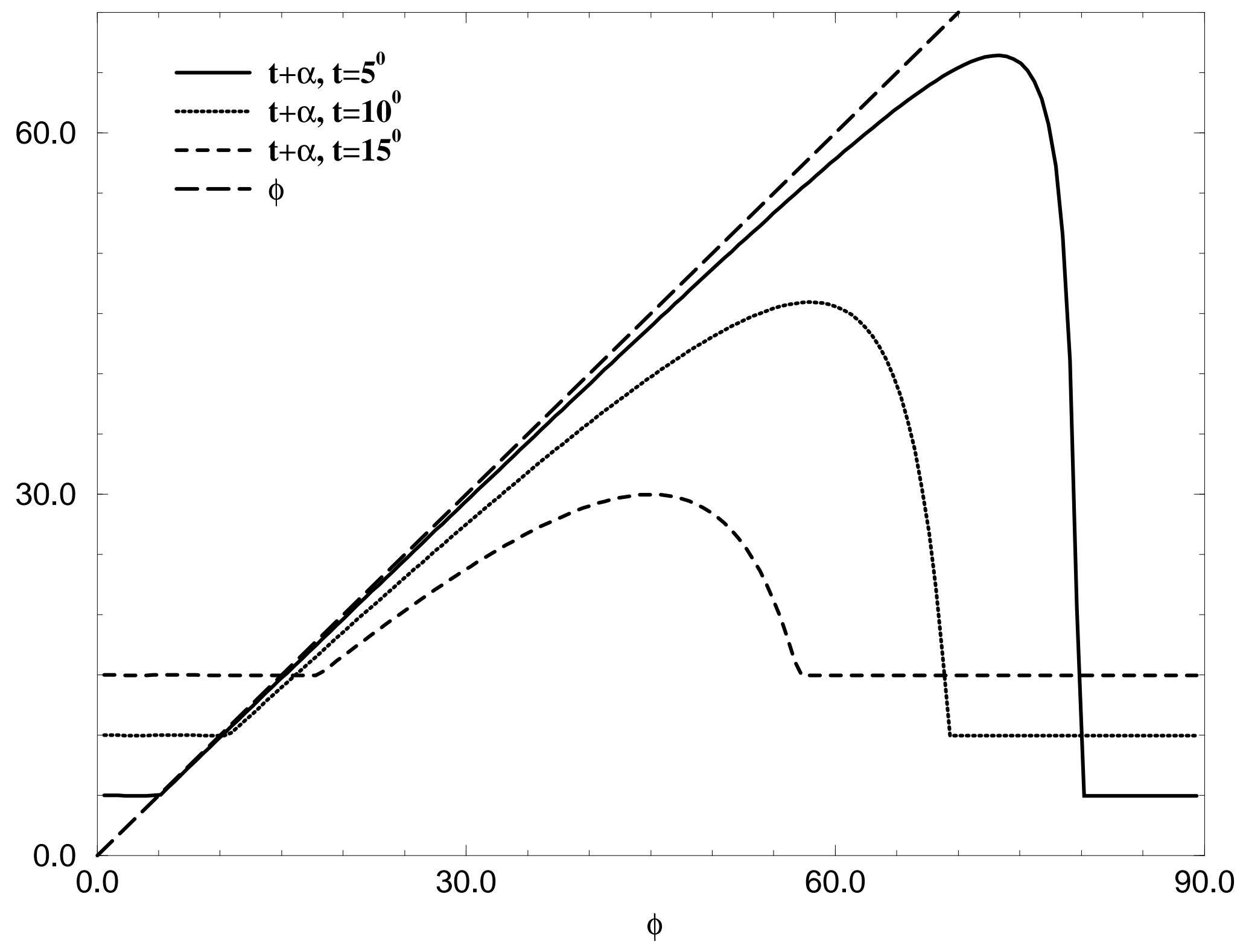




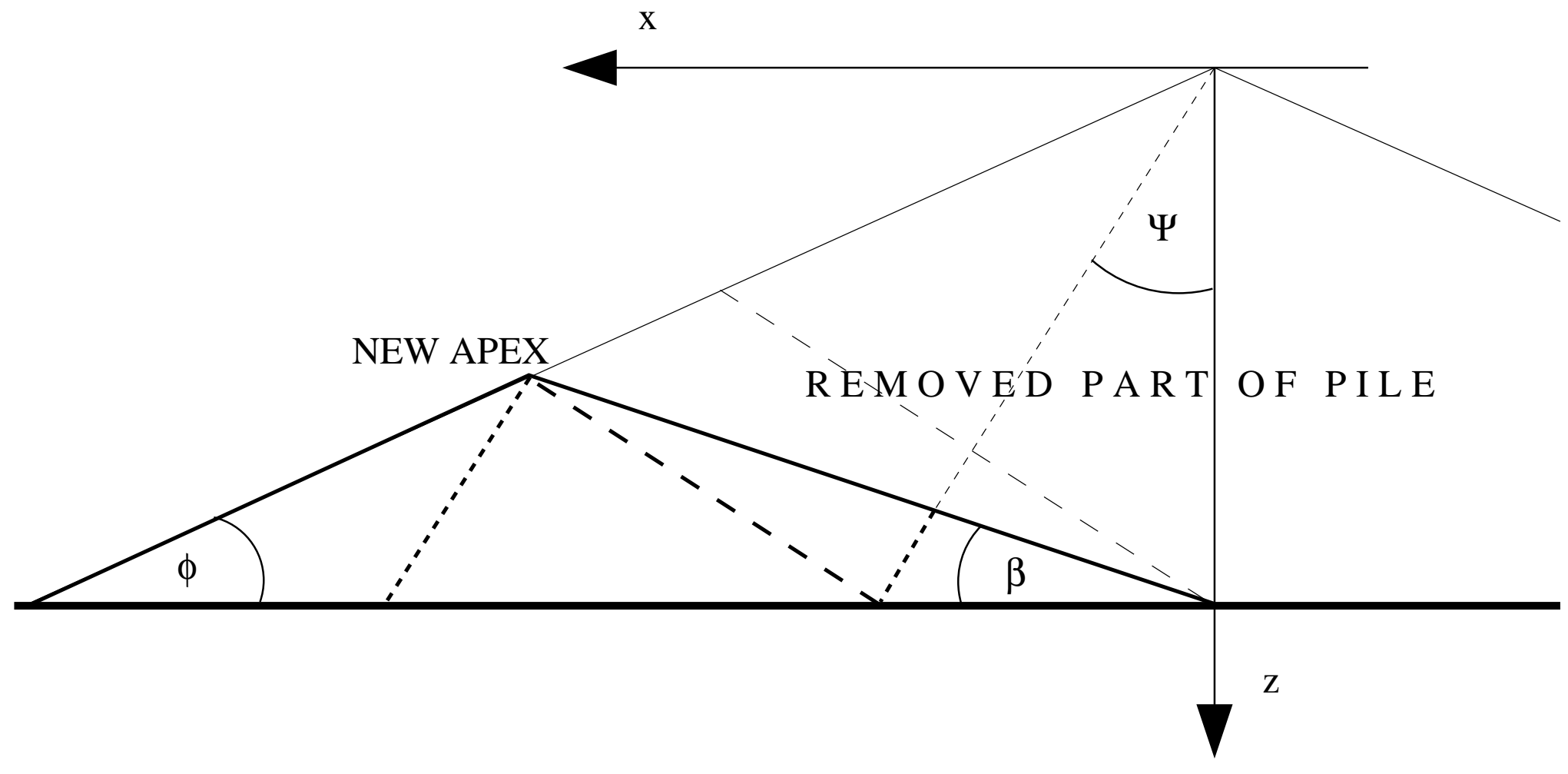




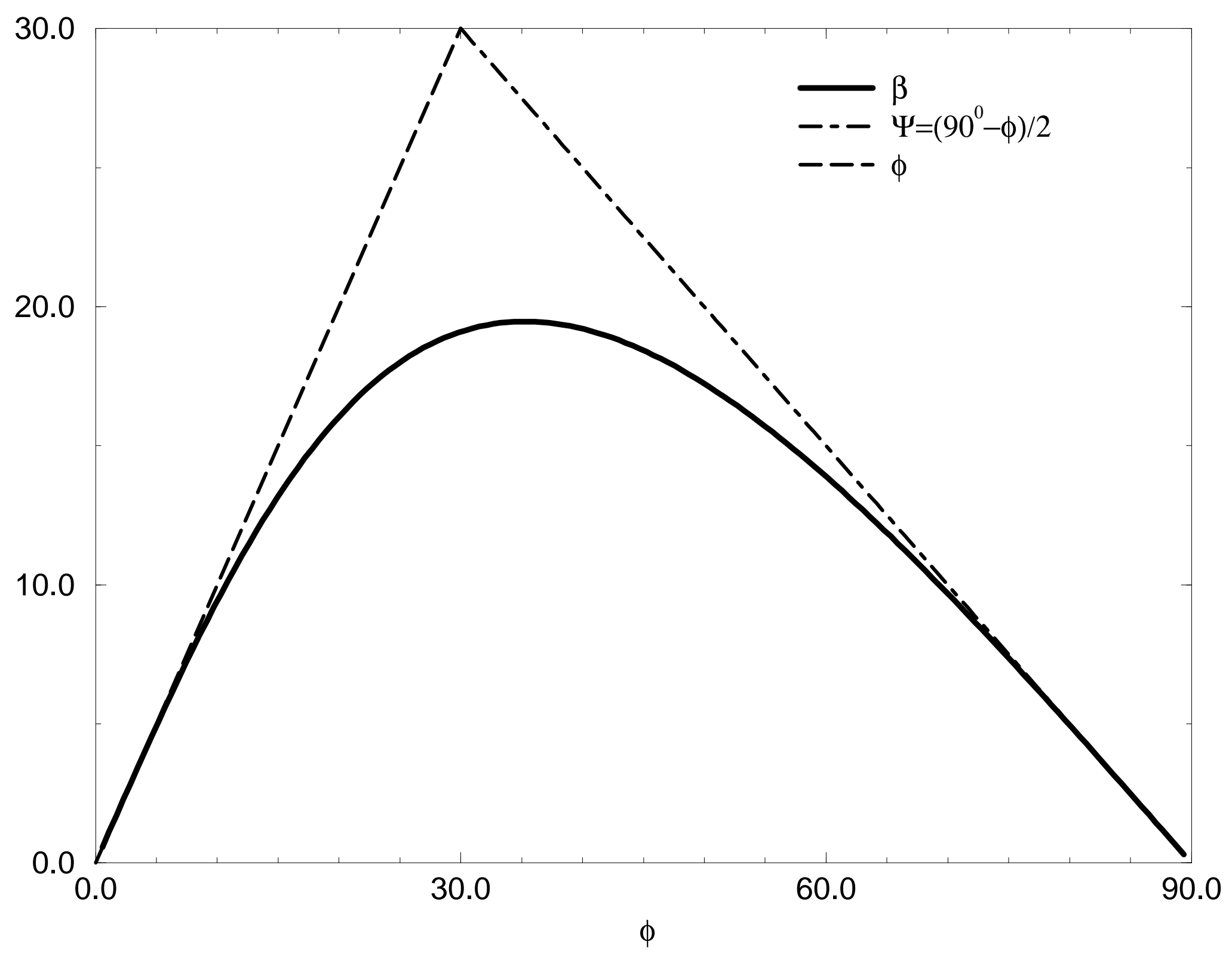




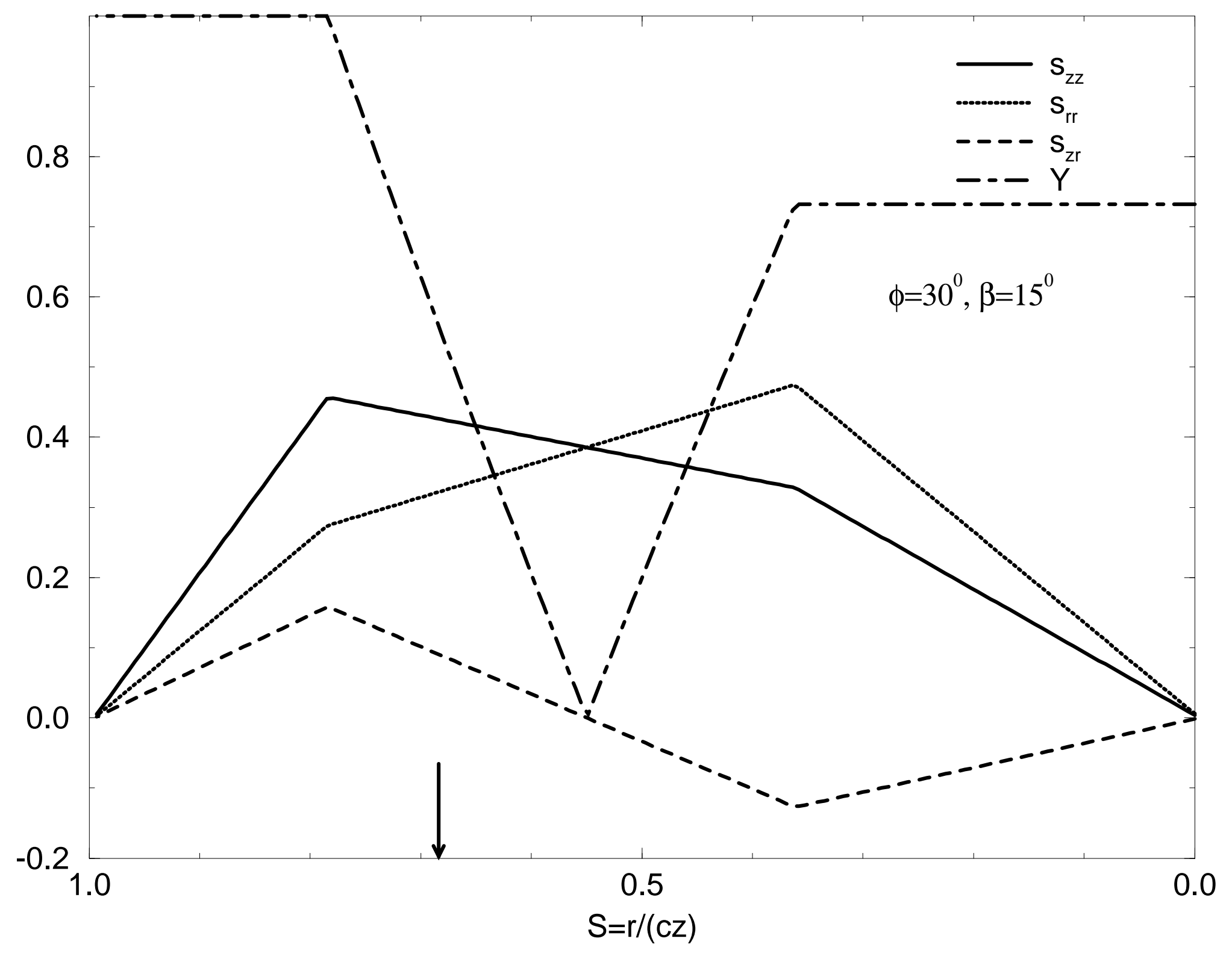




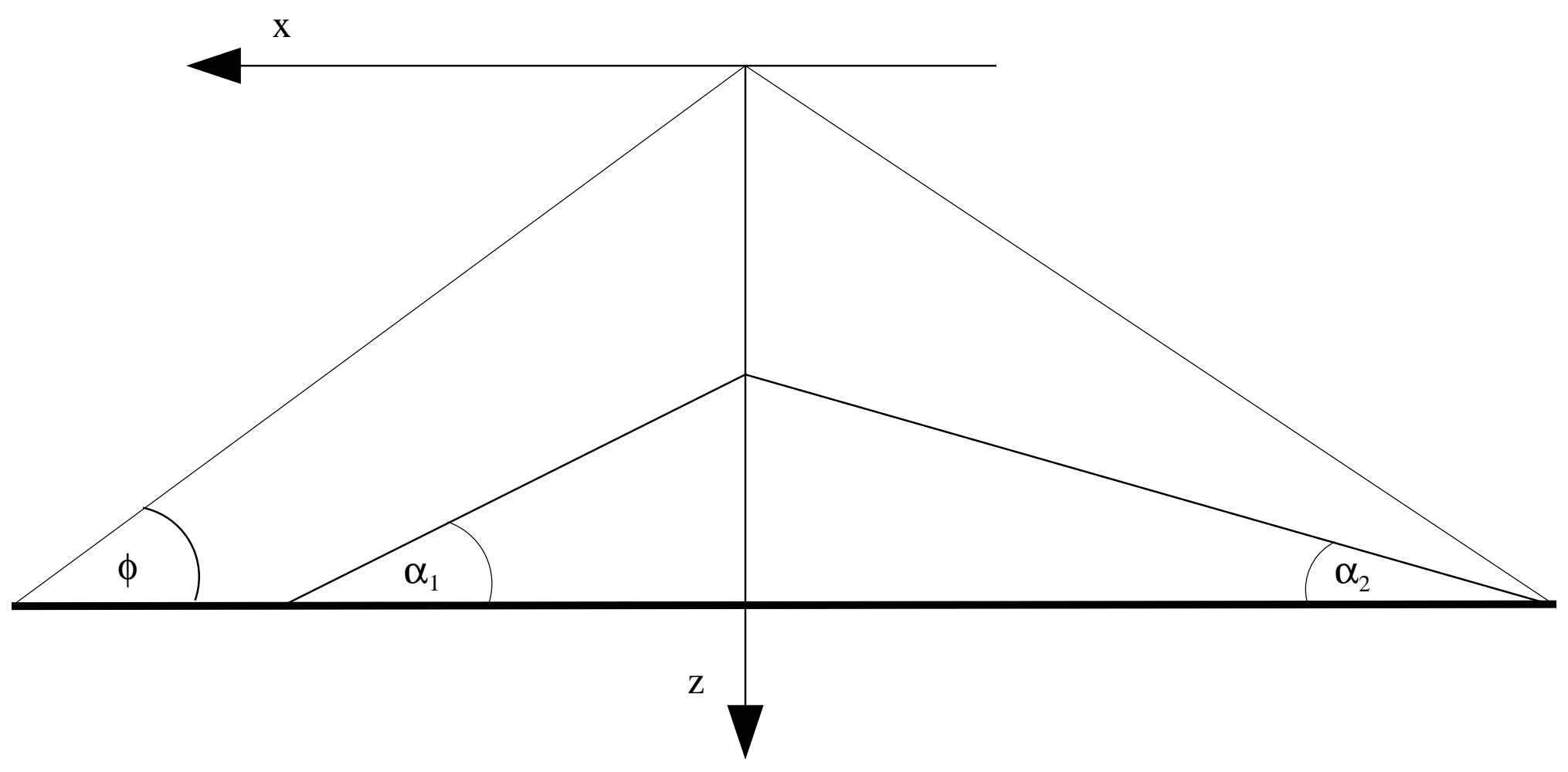




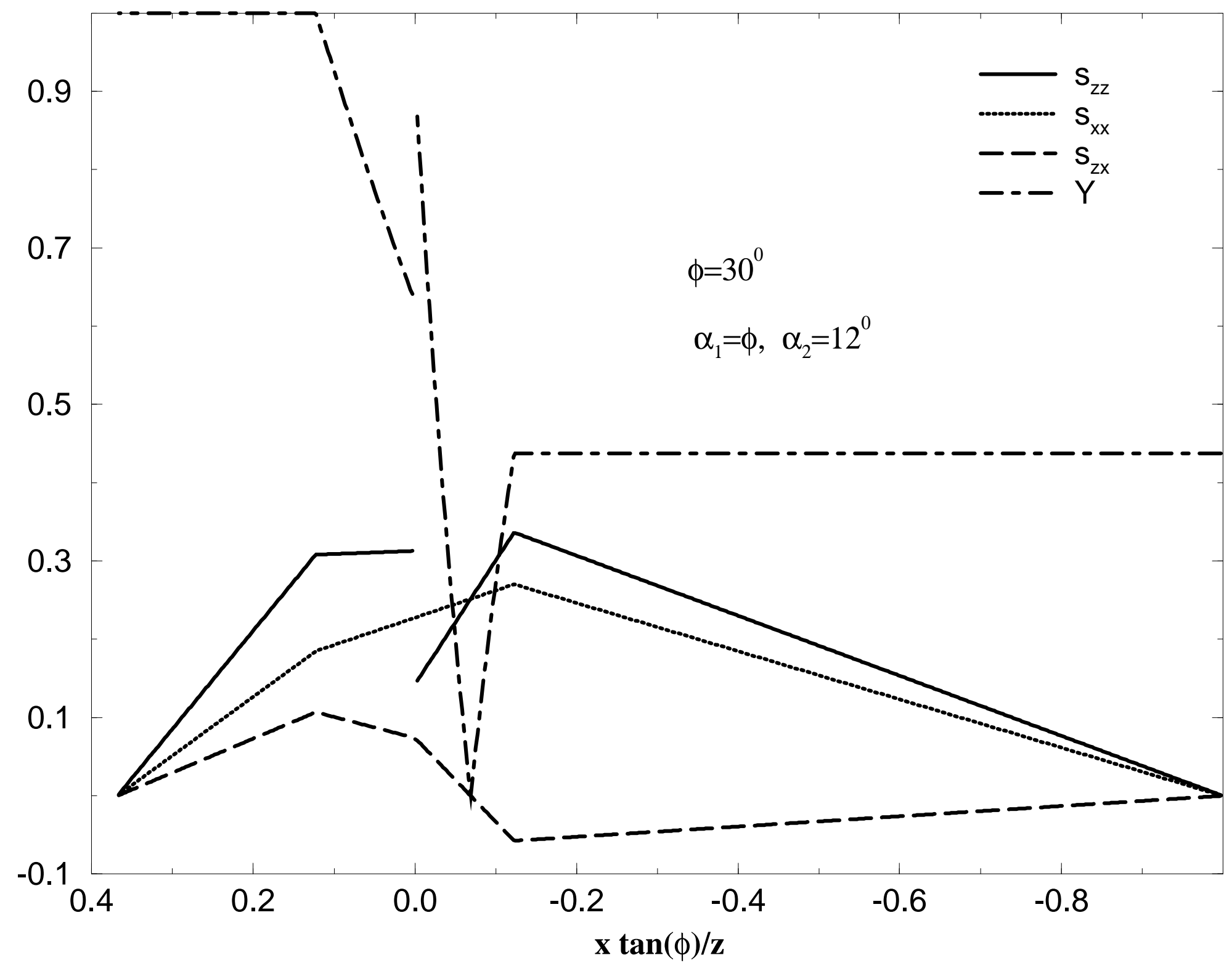




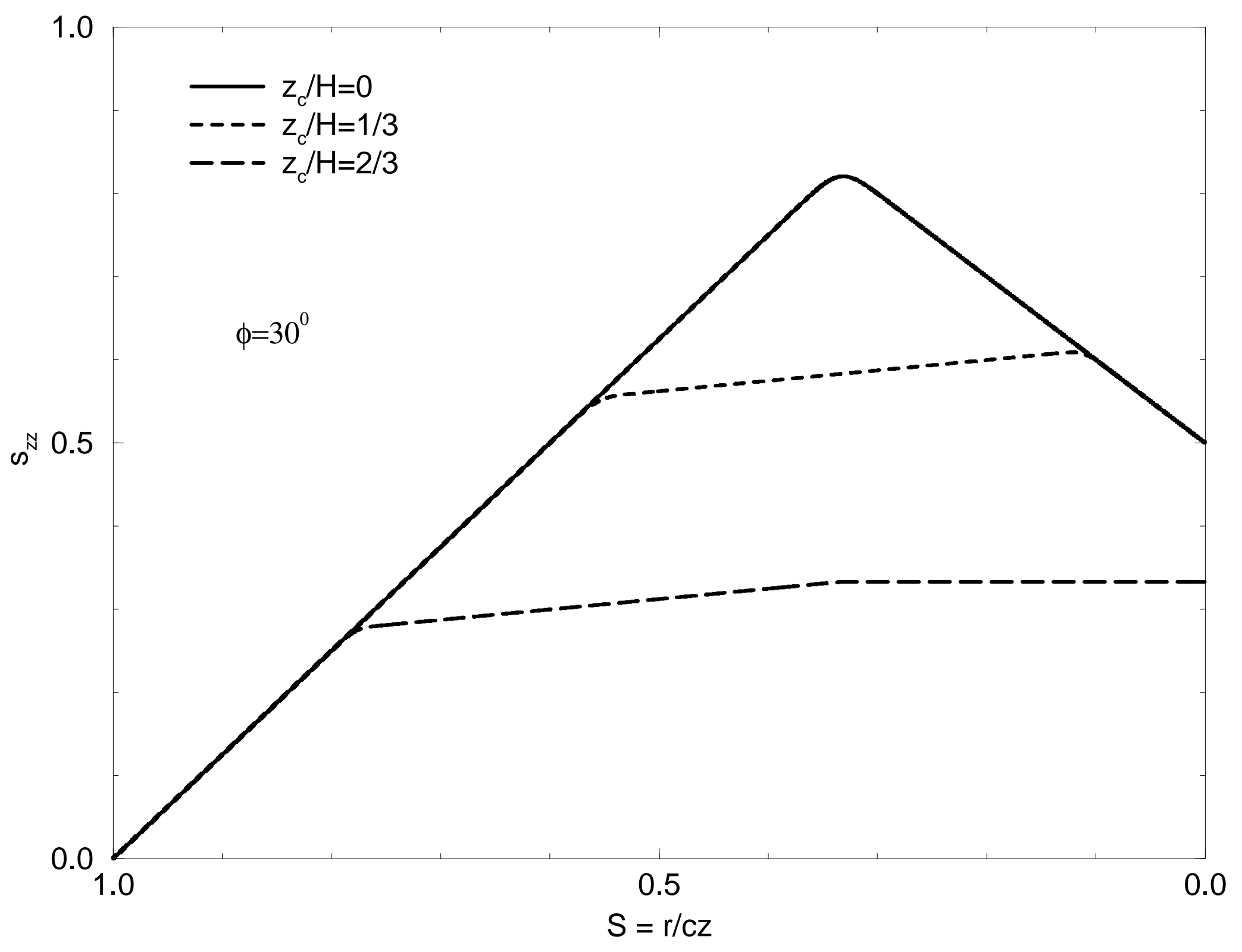

